\title{
Rare-earth elements in the atmosphere of the magnetic chemically peculiar star HD $144897^{\star, \star \star}$
}

\section{New classification of the Nd III spectrum}

\author{
T. Ryabchikova ${ }^{1,2}$, A. Ryabtsev ${ }^{3}$, O. Kochukhov ${ }^{4}$, and S. Bagnulo ${ }^{5}$ \\ 1 Department of Astronomy, University of Vienna, Türkenschanzstrasse 17, 1180 Wien, Austria \\ e-mail: ryabchik@inasan.ru \\ 2 Institute of Astronomy, Russian Academy of Sciences, Pyatnitskaya 48, 119017 Moscow, Russia \\ 3 Institute of Spectroscopy, Russian Academy of Sciences, 142190, Troitsk, Moscow region, Russia \\ 4 Department of Astronomy and Space Physics, Uppsala University Box 515, 75120 Uppsala, Sweden \\ 5 European Southern Observatory, Casilla 19001, Santiago 19, Chile
}

Received 5 April 2006 / Accepted 24 April 2006

\begin{abstract}
Context. The chemically peculiar stars of the upper main sequence represent a natural laboratory for the study of rare-earth elements (REE).

Aims. We want to check the reliability of the energy levels and atomic line parameters for the second REE ions currently available in the literature, and obtained by means of experiments and theoretical calculations.

Methods. We have obtained a UVES spectrum of a slowly rotating strongly magnetic Ap star, HD 144897, that exhibits very large overabundances of rare-earth elements. Here we present a detailed spectral analysis of this object, taking effects of non-uniform vertical distribution (stratification) of chemical elements into account.

Results. We determined the photospheric abundances of 40 ions. For seven elements ( $\mathrm{Mg}, \mathrm{Si}, \mathrm{Ca}, \mathrm{Ti}, \mathrm{Cr}, \mathrm{Mn}, \mathrm{Fe})$, we obtained a stratification model that allows us to produce a satisfactory fit to the observed profiles of spectral lines of various strengths. All the stratified elements but $\mathrm{Cr}$ show a steep decrease in concentration toward the upper atmospheric layers; for $\mathrm{Cr}$ the transition from high to low concentration regions appears smoother than for the other elements. The REEs abundances, which have been determined for the first time from the lines of the first and second ions, have been found typically four dex higher than solar abundances. Our analysis of REE spectral lines provides strong support for the laboratory line classification and determination of the atomic parameters. The only remarkable exception is Nd III, for which spectral synthesis was found to be inconsistent with the observations. We therefore performed a revision of the Nd III classification. We confirmed the energies for 11 out of 24 odd energy levels that were classified previously, and derived the energies for additional 24 levels of Nd III, thereby substantially increasing the number of classified Nd III lines with corrected wavelengths and atomic parameters.
\end{abstract}

Key words. stars: magnetic fields - stars: abundances - stars: chemically peculiar - stars: individual: HD 144897 - atomic data

\section{Introduction}

High overabundances of the rare-earth elements (REE) are the most typical characteristic of the upper main-sequence, magnetic, chemically peculiar (Ap) stars. In the past, REE studies were based on the lines of the first ions, which are rather weak even in the spectra of REE-rich stars with $T_{\text {eff }}>10000 \mathrm{~K}$, which makes blending a severe problem. In normal stars with these temperatures, the lines of singly ionized REE are not visible at all. Therefore, the REE study was limited by the first few most abundant elements, such as $\mathrm{La}, \mathrm{Ce}, \mathrm{Nd}, \mathrm{Sm}$ in some cases, and $\mathrm{Eu}$, which has a few prominent lines in the optical region. The lines of the dominant second ionization stage (REE3) were rarely studied quantitatively because of the lack of atomic data, although their presence in the spectra of Ap stars has been known

* Based on observations collected at the European Southern Observatory, Paranal, Chile (ESO programme No. 68.D-0254).

$\star \star$ Tables 4-7 are only available in electronic form at http://www . edpsciences.org for a long time (see, for example, the pioneering work by Swings (1944) on the REE3 line variations in $\alpha^{2} \mathrm{CVn}$ ).

Significant progress in the laboratory energy level classification provides an opportunity for theoretical calculations of the transition probabilities and other atomic parameters of spectral lines. The most extensive calculations were done by the Belgium group at Mons University, who created a database on rare earths at Mons University - DREAM (Biémont et al. 1999). Other groups, which will be mentioned below, have also performed theoretical calculations of the REE3 atomic structure, which gives a possibility of comparing different results. At present, we have transition probabilities for all but unstable Pm III. Additionally, thanks to efforts of the University of Wisconsin group (references will be given in the corresponding section), high-precision laboratory measurements of the radiative lifetimes and transition probabilities for the first REE ions became available. These data give us a possibility for detailed study of the REE in Ap atmospheres.

However, the first applications of the REE3 atomic data have revealed some inconsistencies between laboratory and 
calculated data (wavelengths, oscillator strengths) and the observed lines in stellar spectra. In particular, wavelengths of a few Nd III lines calculated from the Nd III energy levels (Martin et al. 1978) differ from the stellar ones by up to $0.1 \AA$ and even more (see the line identification list of the HD 122970 spectrum in Ryabchikova et al. 2000). This problem was solved in a study of the laboratory Nd III spectrum by Aldenius (2001), who corrected several energy levels based on high-precision wavelength measurements. However, calculated oscillator strengths give discordant abundance results for some $\mathrm{Nd}$ III lines in $\mathrm{HgMn}$ stars (Dolk et al. 2002).

The Ap stars provide a perfect natural laboratory for REE study. First, they have extreme overabundances of these elements, therefore their spectra contain a large number of spectral lines of both ionization stages. Second, magnetic splitting may give extra information needed to verify line classification. And third, rapidly oscillating roAp stars have the outstanding pulsational characteristic that in most stars only REE lines show large radial velocity pulsation amplitudes (Savanov et al. 1999; Kochukhov \& Ryabchikova 2001a,b), therefore we can doublecheck whether a certain spectral feature belongs to REEs by looking at its pulsational characteristics.

We chose HD 144897 as a template. This star has a surface magnetic field $\left\langle B_{\mathrm{s}}\right\rangle$ varying between 8.5 and $9.5 \mathrm{kG}$ over the period $48.43 \mathrm{~d}$. (Mathys et al. 1997). This field is strong enough to produce clear Zeeman splitting of the spectral lines in the unpolarized spectrum of the slowly rotating star, and not too large to produce an overlapping of the Zeeman components from nearby spectral lines. The quick look at the HD 144897 spectrum shows resolved Zeeman split features at the positions of strong Pr III and Nd III lines. Furthermore, HD 144897 has never been studied for abundances, therefore our analysis will provide additional information for Ap-star chemistry.

Our paper is structured as follows. Observations and data reduction are described in Sect. 2, and the determination of the fundamental stellar parameters is presented in Sect. 3. The results of the magnetic spectrum synthesis in the homogeneous, as well as in chemically stratified, atmosphere are given in Sect. 4. Section 5 revises classification of the Nd III spectrum. Discussion and conclusions are given in Sects. 6 and 7, respectively.

\section{Observations and data reduction}

High-resolution, high signal-to-noise-ratio UVES spectra of HD 144897 were obtained with the UVES instrument of the ESO VLT on 26 February 2002 within the context of program 68.D-0254. The UVES instrument is described by Dekker et al. (2000). The observations were carried out using both available dichroic modes. The detailed log of the observations is given in Table 1. In both the blue arm and the red arm, the slit width was set to $0.5^{\prime \prime}$, for a spectral resolution of about 80000 . The slit was oriented along the parallactic angle, in order to minimize losses due to atmospheric dispersion. Almost the full wavelength interval from 3030 to $10400 \AA$ was observed except for a few gaps, the largest of which was at 5760-5835 $\AA$ and 8550-8650. . In addition, there are several small gaps, about $1 \mathrm{~nm}$ each, due to the lack of overlapping between the échelle orders in the $860 \mathrm{U}$ setting.

The UVES data were reduced with the automatic pipeline described in Ballester et al. (2000). For all settings, science frames are bias-subtracted and divided by the extracted flatfield, except for the 860 setting, where the 2D (pixel-to-pixel)
Table 1. Log of UVES observations of HD 144897.

\begin{tabular}{ccc}
\hline \hline Date & UT & Setting $(\mathrm{nm})$ \\
\hline $2002-02-26$ & $08: 01: 25$ & 346 \\
$2002-02-26$ & $08: 10: 26$ & 346 \\
$2002-02-26$ & $08: 01: 24$ & 580 \\
$2002-02-26$ & $08: 10: 32$ & 580 \\
$2002-02-26$ & $08: 25: 07$ & 437 \\
$2002-02-26$ & $08: 25: 07$ & 860 \\
\hline
\end{tabular}

flat-fielding is used, in order to correct for the fringing better. Because of the high flux of the spectra, we used the UVES pipeline average extraction method. All spectra were normalized to the continuum with the help of an interactive procedure, which employed either a low-degree polynomial or a smoothing spline function.

\section{Fundamental parameters}

The initial estimate of the effective temperature and surface gravity of HD 144897 was derived using photometric data in the Geneva system. The measurements were taken from the online catalogue maintained at the Geneva Observatory (Burki et al. 2005) ${ }^{1}$. The Hipparcos parallax, $\pi=4.77 \pm 1.28$ mas, (Perryman et al. 1997) and galactic coordinates of the star suggest that its reddening is significant. From a variety of sources (Lucke 1978; Hakkila et al. 1997; Schlegel et al. 1998), we obtained $E(B-V)$ in the range 0.13 to 0.48 . Significant reddening is confirmed by the presence of very strong $\mathrm{NaD}$ interstellar absorption lines. In this situation, the best approach is to make use of the reddening-free $X, Y$ parameters of the Geneva photometry. Using the calibration of Künzli et al. (1997), we determined $T_{\text {eff }}=12051 \mathrm{~K}$ and $\log g=4.34$. Subsequent correction for the anomalous flux distribution of Ap stars (Hauck \& Künzli 1996) yields $T_{\text {eff }}=11100 \mathrm{~K}$. Stellar parameters were further adjusted to fit the hydrogen $\mathrm{H} \alpha$ and $\mathrm{H} \beta$ lines. Finally, we obtained $T_{\text {eff }}=11250 \mathrm{~K}$ and $\log g=4.0$. Model atmosphere of HD 144897 was calculated with the ATLAS9 code (Kurucz 1993), using ODF with 10 times solar metallicity and microturbulent velocity $4 \mathrm{~km} \mathrm{~s}^{-1}$ to mimic the enhancement of line opacity due to magnetic intensification.

We estimated the averaged surface magnetic field $\left\langle B_{\mathrm{s}}\right\rangle$ by measuring the distance between the resolved Zeeman components of either pure doublets (Fe II 3303.46, 6149.48 $\AA$, Cr II $3421.20 \AA$ ) or pseudo-triplets (Fe I $4260.47 \AA$, Nd III 6145.07 ̊, Cr II 6231.68, 6275.87 ̊). Zeeman components were approximated by the sum of Gaussians. The results for both groups are similar, and the final value is $\left\langle B_{\mathrm{s}}\right\rangle=8.79 \pm 0.10 \mathrm{kG}$. Our observations $(\mathrm{HJD}=2452331.83$ ) correspond to a rotation phase 0.13 for the ephemeris given in Mathys et al. (1997), and the derived $\left\langle B_{\mathrm{s}}\right\rangle$ is fully consistent with their magnetic curve for HD 144897.

The projected rotational velocity was estimated using the rather weak magnetically insensitive line Fe II $6586.70 \AA$ (mean Landé factor $\left.g_{\mathrm{eff}}=0.02\right)$ and medium intensity Fe II $4491.40 \AA$ $\left(g_{\text {eff }}=0.4\right)$. Both lines indicate $v_{\mathrm{e}} \sin i \approx 4 \mathrm{~km} \mathrm{~s}^{-1}$, which seems a little too high to clearly reproduce the visible Zeeman structure of many weak lines. A better fit of the synthetic spectrum to the observations is provided by $v_{\mathrm{e}} \sin i=3 \mathrm{~km} \mathrm{~s}^{-1}$, therefore we adopted this value in all the abundance analysis calculations.

\footnotetext{
${ }^{1}$ http://obswww. unige.ch/gcpd/ph13.html
} 


\section{Abundance analysis}

A very important part of all abundance calculations is accurate atomic parameters of spectral lines. Most atomic data in our study are taken from the VALD database (Kupka et al. 1999), which has been updated recently to include the new line lists: Ti II (Pickering et al. 2001), Cr II, Fe II, Co II (Raassen \& Uylings 1998), Mn II (Kling \& Griesmann 2000; Kling et al. 2001). The DREAM REE line database (Biémont et al. 1999) is also made accessible via the VALD extraction procedures. For the second REE ions, Landé factors were calculated by Quinet et al. (2004). For Sr, Y, Zr, Ba, Re Landé factors were taken from the "Atomic Energy Levels" tables by Moore (1971). For light elements O, $\mathrm{Mg}$, and Si Landé factors were calculated in the LS approximation. For Si II, new experimental data on the Stark broadening and transition probabilities are available (Wilke 2003), which allows us to increase the number of Si II lines included in the abundance analysis. The new Si II oscillator strengths are smaller by 0.05 dex on average compared to the values in VALD (see also Ryabchikova et al. 2005). This difference does not influence the results of both standard and stratification analysis. Detailed description of the REE atomic parameters will be given below.

\subsection{Spectrum synthesis}

Computations of the synthetic spectra of HD 144897 were carried with the help of the magnetic spectrum synthesis code SYNTHMAG_FAST, which represents an improved version of the program described by Piskunov (1999). In this spectrum synthesis approach, the polarized radiative transfer equation is solved numerically for seven values of the angle between the line of sight and the normal to the stellar surface. Resulting intensity spectra are integrated for a given rotational velocity. SYNTHMAG_FAST uses a simplified model of the stellar magnetic field geometry with a homogeneous surface distribution of both the field strength and field orientation. Although not suitable for detailed analysis of the polarization spectra, this model is adequate for the purpose of fitting magnetically enhanced and split lines in the Stokes $I$ spectrum. In our study SYNTHMAG_FAST was used to calculate spectra both for homogeneous and stratified vertical distribution of chemical abundances. The modelling of the lines with different Zeeman patterns requires a mean inclination of the magnetic vector to the stellar surface $\sim 50-60^{\circ}$ to reproduce relative intensities of the $\pi$ and $\sigma$ components.

The final abundances calculated for the magnetic field $\left\langle B_{\mathrm{s}}\right\rangle=8.8 \mathrm{kG}$ are presented in Table 2 . The full list of the measured lines is available in electronic form only (Table 5 of Online material). In Table 2, abundances derived for HD 144897 are compared with the recently published abundances of the two stars with similar effective temperatures: HD 170973 (Kato 2003) and HD 116458 (Nishimura et al. 2004). The recent solar photospheric abundances (Asplund et al. 2005) are given in the last column. With a few exceptions abundances in all 3 Ap stars are similar, in particular, for the REE.

\subsection{Abundance stratification}

Preliminary analysis of the HD 144897 spectrum revealed the presence of $\mathrm{Fe}$ lines in three ionization stages, with plenty of strong high-excitation Fe II lines. It was impossible to fit them with the same abundance. Also, the lines of Si II and Si III are present, and again it is not possible to explain them with the same abundance. Therefore, we performed a stratification analysis of a few chosen elements that have enough spectral lines
Table 2. Element abundances in the Ap star HD 144897 based on $n$ spectral lines. For elements with the stratification analysis, an abundance that provides the best fit to $n$ line profiles in a homogeneous atmosphere is given. Abundances in the atmospheres of the Ap stars HD 170973 and HD 116458 and in the solar photosphere (Asplund et al. 2005) are given for comparison. Results obtained from only one line are marked by “:”.

\begin{tabular}{|c|c|c|c|c|c|}
\hline \multirow[t]{2}{*}{ Ion } & \multicolumn{2}{|c|}{$\overline{\text { HD } 144897}$} & \multirow{2}{*}{$\begin{array}{l}\text { HD 170973 } \\
\log \left(N / N_{\text {tot }}\right)\end{array}$} & \multirow{2}{*}{$\begin{array}{l}\text { HD 116458 } \\
\log \left(N / N_{\text {tot }}\right)\end{array}$} & \multirow{2}{*}{$\begin{array}{c}\text { Sun } \\
\log \left(N / N_{\text {tot }}\right)\end{array}$} \\
\hline & $\log \left(N / N_{\text {tot }}\right)$ & $n$ & & & \\
\hline OI & -4.0 & 3 & -2.92 & -3.71 & -3.38 \\
\hline $\mathrm{Mg}$ & -5.6 & 3 & -4.87 & -4.80 & -4.51 \\
\hline $\mathrm{Si}$ & -3.8 & 11 & -3.30 & -4.27 & -4.53 \\
\hline $\mathrm{Ca}$ & -6.0 & 5 & -5.36 & & -5.73 \\
\hline $\mathrm{Ti}$ & -6.4 & 16 & -4.56 & -6.53 & -7.14 \\
\hline $\mathrm{Cr}$ & -4.3 & 23 & -4.48 & -4.54 & -6.40 \\
\hline $\mathrm{Mn}$ & -5.3 & 8 & -5.40 & -5.72 & -6.65 \\
\hline $\mathrm{Fe}$ & -3.5 & 31 & -2.91 & -3.72 & -4.59 \\
\hline CoI & $-4.67(12)$ & 3 & & -3.59 & -7.12 \\
\hline Co II & $-4.51(32)$ & 7 & & -3.67 & -7.12 \\
\hline Sr II & $-7.45(55)$ & 4 & -6.18 & -9.06 & -9.12 \\
\hline $\mathrm{Y}_{\text {II }}$ & $-7.70(09)$ & 3 & & -7.69 & -9.83 \\
\hline Zr II & $-7.38(15)$ & 8 & -7.45 & & -9.45 \\
\hline Ba II & $-9.0:$ & 1 & -9.42 : & -9.92 : & -9.87 \\
\hline La II & $-7.43(12)$ & 4 & -7.92 : & -8.00 : & -10.91 \\
\hline Ce II & $-6.69(20)$ & 10 & -6.87 & -7.34 & -10.46 \\
\hline Ce III & $-6.64(18)$ & 8 & & & -10.46 \\
\hline Pr II & $-6.60(14)$ & 2 & -7.19 & -7.30 & -11.33 \\
\hline Pr III & $-6.69(14)$ & 14 & -6.87 & -7.32 & -11.33 \\
\hline Nd II & $-6.45(12)$ & 9 & -6.48 & & -10.59 \\
\hline Nd III & $-6.45(20)$ & 13 & -6.63 & -7.26 & -10.59 \\
\hline Sm II & $-6.98(21)$ & 4 & -7.07 & & -11.03 \\
\hline Sm III & $-6.92(20)$ & 10 & & & -11.03 \\
\hline Eu II & $-7.75(20)$ & 4 & -7.77 & -7.94 & -11.52 \\
\hline Eu III & $-6.32(23)$ & 6 & & -6.70 & -11.52 \\
\hline Gd II & $-6.95(18)$ & 13 & -7.17 : & -7.47 & -10.92 \\
\hline Gd III & $-6.60(14)$ & 2 & & & -10.92 \\
\hline Tb II & $-7.83(10)$ & 3 & & & -11.76 \\
\hline Tb III & $-7.92(22)$ & 6 & -7.96 & & -11.76 \\
\hline Dy II & $-7.12(22)$ & 11 & -7.17 & -7.25 : & -10.90 \\
\hline Dy III & $-6.99(39)$ & 6 & -7.13 & -7.93: & -10.90 \\
\hline Ho II & $-8.00(10)$ & 3 & & & -11.53 \\
\hline Ho III & $-8.08(16)$ & 6 & -7.92 & & -11.53 \\
\hline Er II & $-7.55(14)$ & 2 & $-7.54:$ & & -11.11 \\
\hline Er III & $-7.21(14)$ & 7 & -7.79 & & -11.11 \\
\hline Tm II & $-8.12(20)$ & 3 & & & -12.04 \\
\hline Tm III & $-7.70(20)$ & 3 & & & -12.04 \\
\hline YbII & -7.50 : & 1 & & & -10.96 \\
\hline Yb III & $-7.50:$ & 1 & & & -10.96 \\
\hline Lu II & $-8.60:$ & 1 & & & -11.98 \\
\hline$T_{\text {eff }}$ & $11250 \mathrm{~K}$ & & $10750 \mathrm{~K}$ & $10300 \mathrm{~K}$ & $5777 \mathrm{~K}$ \\
\hline $\log g$ & 3.70 & & 3.50 & 3.81 & 4.44 \\
\hline$\left\langle B_{\mathrm{s}}\right\rangle(\mathrm{kG})$ & 8.8 & & 0.0 & 4.7 & \\
\hline$v_{\mathrm{e}} \sin i\left(\mathrm{~km} \mathrm{~s}^{-1}\right)$ & 3.0 & & 4.0 & 8.0 & \\
\hline
\end{tabular}

of different strength, ionization (where possible), excitation, and sensitivity to Stark effect, and that lie on both sides of the Balmer jump (BJ). For Mn, hyperfine splitting was taken into account using the data from Holt et al. (1999). The same step function approximation of the abundance distribution that was adopted by Ryabchikova et al. (2005) in their analysis of HD 204411 was used here. The details of stratification analysis are given in the cited paper. The only difference is that we used magnetic spectrum synthesis for HD 144897 instead of a regular spectrum synthesis for HD 204411. Stratification calculations were performed for $\mathrm{Mg}, \mathrm{Si}, \mathrm{Ca}, \mathrm{Ti}, \mathrm{Cr}, \mathrm{Mn}$, and Fe. First, we derived a mean abundance that gives the best fit for all chosen line profiles, treated the same way as in stratification procedure, but with a chemically homogeneous atmosphere. These homogeneous abundances are presented in Table 2 together with the number of lines used in the analysis, and they were used as an initial guess for the stratification study. We also calculated the mean deviation between the observed and calculated line profiles, which may be considered as a measure of the standard error. The obtained element distributions are shown in Fig. 1. An example of the fits to a sample of Fe line profiles representing 


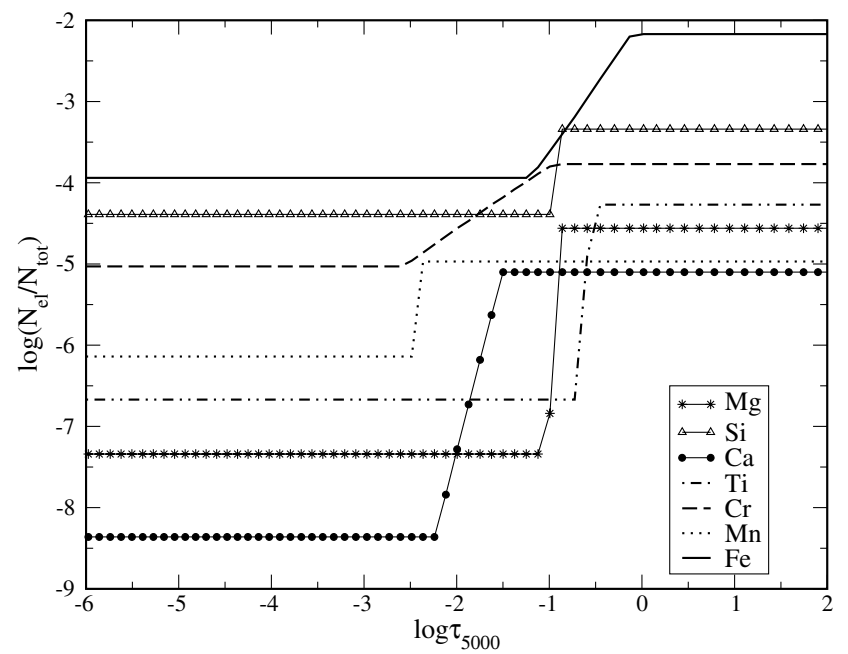

Fig. 1. Abundance stratification in the atmosphere of HD 144897.

a variety of Zeeman patterns is given in Fig. 2. We do not expect to get a better fit of the Zeeman components due to the crude magnetic geometry used in the magnetic spectrum synthesis. The improvement of the fit with the stratified abundances over the one with the homogeneous element distribution is also shown in Fig. 10.

The Co II lines are very strong in HD 144897, especially in the spectral region below BJ. They indicate an overall Co abundance of $\log \left(\mathrm{Co} / N_{\text {tot }}\right)=-4.2:-5.0$, i.e. more than 3 dex above the solar photospheric abundance. However, the lack of hyperfine splitting $(h f s)$ data for Co II lines does not allow us to perform a detailed analysis for this element.

All stratified elements but $\mathrm{Cr}$ show a steep decrease in concentration towards the upper atmospheric layers. The position of the abundance jump varies from $\log \tau_{5000} \approx-1(\mathrm{Mg}, \mathrm{Si}, \mathrm{Ti}$, and $\mathrm{Fe}$ ) to $-2.5(\mathrm{Mn})$. The $\mathrm{Mg}$ distribution in HD 144897 differs from the distribution derived in HD 204411 (Ryabchikova et al. 2005) and in HD 133792 (Kochukhov et al. 2006), where a slow increase in the $\mathrm{Mg}$ abundance towards the upper atmosphere was obtained. We cannot consider our present results for $\mathrm{Mg}$ as conclusive because of the small number of $\mathrm{Mg}$ lines available for HD 144897. More stars in a wide temperature range should be investigated to get a better picture of the abundance distribution variations with effective temperature and other stellar parameters. In HD 144897 the abundance jumps for $\mathrm{Cr}, \mathrm{Mn}$, and $\mathrm{Fe}$ are not very large, $\sim 1-2$ dex. The jump is larger for $\mathrm{Fe}$ than for $\mathrm{Cr}$ and Mn. As demonstrated by Shulyak et al. (2004), similar Fe distribution in the hotter star CU Vir $(12750 \mathrm{~K})$ leads to better modelling of the observed energy distribution, in particular, the $\lambda 5200 \AA$ depression. Similar Ca and $\mathrm{Cr}$ distributions, but with slightly smaller abundance jumps, were derived in the atmospheres of cooler and more evolved stars HD 204411 (8400 K) and HD 133792 (9400 K). Ca has the largest jump in all three stars. The Si abundance jump is smaller than in HD 204411 and HD 133792. It is roughly comparable to the Si distribution in the slightly hotter star HD 10221 (Glagolevskij et al. 2005).

For all elements except $\mathrm{Mg}$ and $\mathrm{Ca}$, abundances are never below solar over the part of the atmosphere where we can consider our results to be reliable. This atmospheric part is limited by the line-formation sensitivity to temperature and pressure. We cannot say much about abundances above $\log \tau_{5000}=-4$.

\subsection{Rare-earth elements}

HD 144897 is the second object after the famous Przybylski's star (HD 101065) for which abundances of all REEs but Pm are derived, and the first star in which abundances for most REEs are derived from the lines of the first and second ions. For odd isotopes, hyperfine splitting is known and can be taken into account. However, in the presence of a magnetic field, the coupling between the Zeeman and $h f s$ components changes the shape of the spectral line and should be taken into account in spectral synthesis. However, we noticed that in most cases the line profiles are fitted reasonably well with the pure Zeeman splitting. The lines of the first ions of the REE are rather weak in the HD 144897 spectrum, and the derived abundance should not be affected much by the $h f s$ effects. Consequently, we did not use the $h f s$ data in our analysis, with the exception of Pr II, Eu II, and Lu II. Below we briefly discuss each element.

When no specific reference is given for atomic data, it means that all relevant parameters are extracted from VALD (Kupka et al. 1999).

Lanthanum. The oscillator strengths and $h f s$ constants for La II lines are taken from Lawler et al. (2001a). Two lines of La III at $\lambda 3171.69$ and 3517.16 are seen in our spectrum, but the lines are rather broad and are obviously affected by $h f s$, which is unknown.

Cerium. The oscillator strength for Ce II and Ce III lines were taken from Palmeri et al. (2000) and from Biémont et al. (2002a), respectively. All lines are nicely reproduced with the adopted magnetic field. Figure 3 shows a fit of the synthesized Ce III $3470.92 \AA$ line to the observed one. Most Ce lines in HD 144897 are represented well with the available atomic data.

Praseodymium. Atomic data from VALD and $h f s$ constants from Ginibre (1989) were used in the Pr II analysis, which was based on two relatively weak lines at $\lambda \lambda$ 4206.72, 5322.77. Other Pr II lines are in blends. For Pr III lines, new calculations by one of us (AR) based on the extended energy levels given by Wyart et al. (2006) were applied. New calculations contain transition probabilities for the $4 \mathrm{f}^{34} \mathrm{~F}$ term, which is missing in the DREAM list (Biémont et al. 2001c). However, a number of intermediate strength lines from this term are observed in our spectrum. In general, a difference between DREAM's and the present oscillator strengths for 10 lines in common does not exceed 0.08 dex. Calculated Landé factors represent the observed Zeeman patterns nicely (see Fig. 4 for Pr III $5844.41 \AA$ A).

Neodymium. Recent experimental oscillator strengths (Den Hartog et al. 2003) were used to derive abundances from Nd II lines. Analysis of the Nd III lines met with difficulties, because we did not find the number of spectral lines which should be visible according to DREAM data. For some spectral lines, for example, $\lambda \lambda$ 3590.32, $4903.24 \AA$ the theoretical Zeeman pattern deviates from the observed one (Figs. 5, 10). The new Nd III classification will be considered in Sect. 5 . We based our abundance determinations on the lines whose Zeeman patterns are reproduced well by the Landé factors given in three independent sets of theoretical calculations: Bord (2000), Zhang et al. (2002b), and the present study (Sect. 5). An example of the fit for the Nd III $5845.02 \AA$ line is shown in Fig. 4.

Samarium. Accurate experimental data are available now for $958 \mathrm{Sm}$ II lines (Lawler et al. 2006), so that we used them in our analysis. Note, for the 4 lines studied here, the data by Lawler et al. agree perfectly with the line parameters included in VALD. For Sm III lines, oscillator strengths are taken from Biémont et al. (2003). The authors give 3 sets of data: experimental transition probabilities for a limited number of lines, 


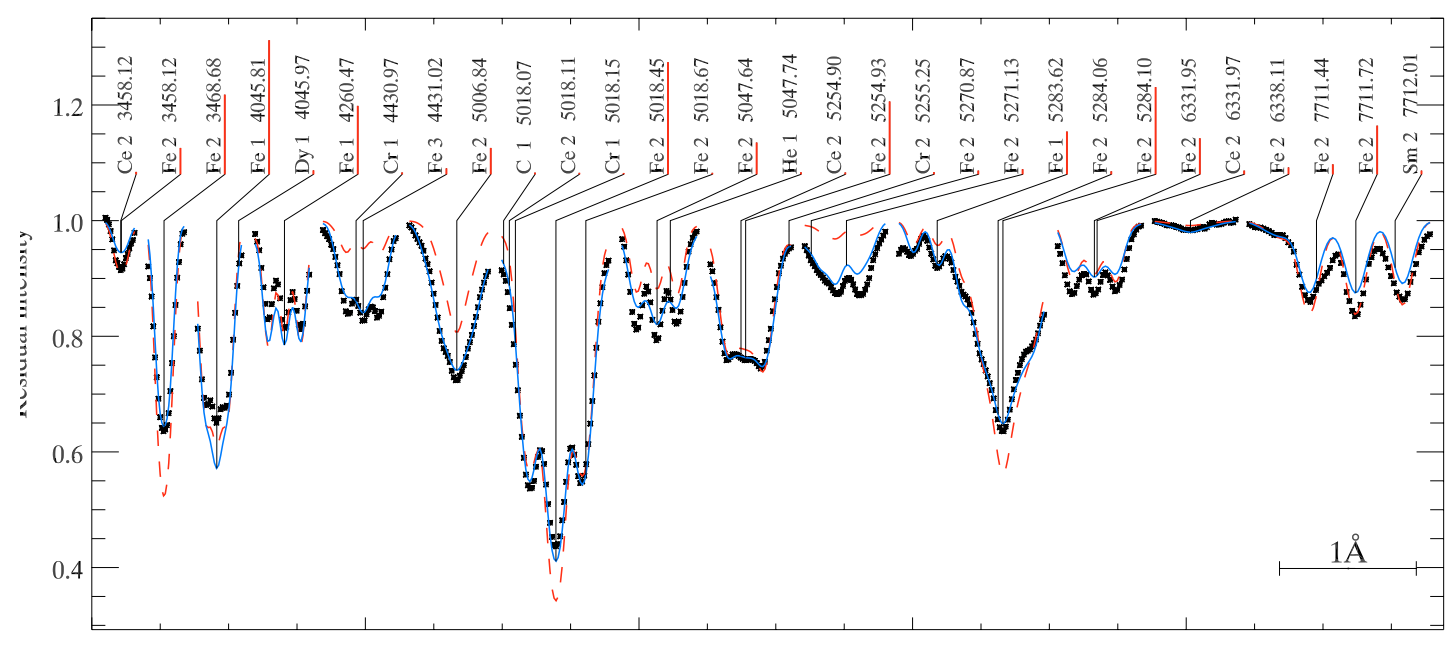

Wavelength

Fig. 2. A comparison between the observed Fe line profiles and calculations with stratified abundance distribution (solid line) and with homogeneous abundances (dashed line) from Table 2.

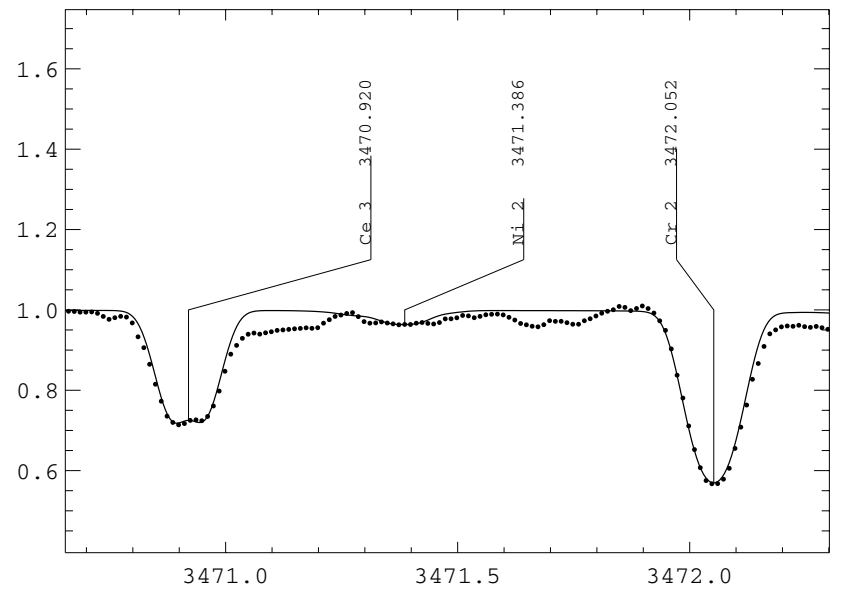

Fig. 3. The Ce III $3470.92 \AA$ line in the spectrum of HD 144897 (dots). Spectral synthesis is shown by a solid line.

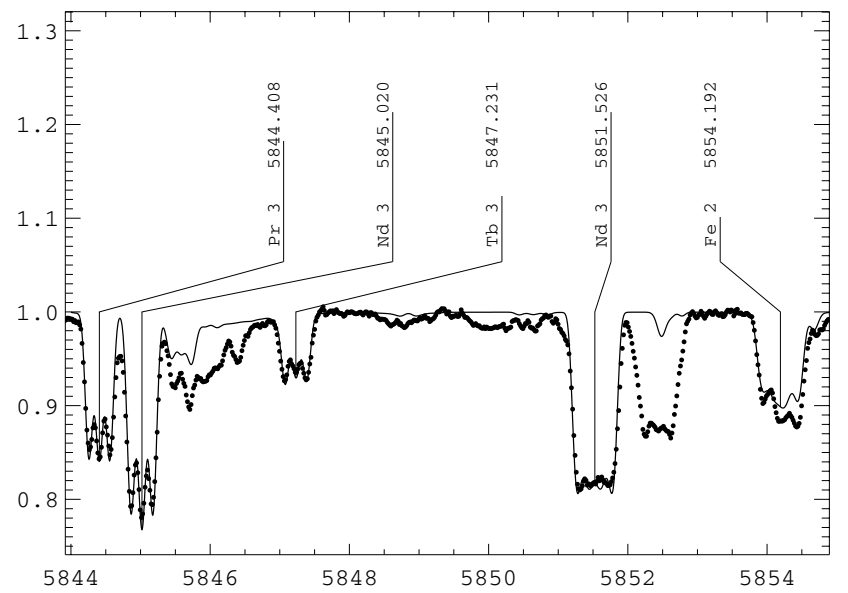

Fig. 4. Examples of the fit for Pr III, Nd III, and Tb III lines (solid line) to the observed HD 144897 spectrum (dots).

relativistic Hartree-Fock (HF) calculations combined with the experimental branching ratios (BR), and HF calculations with the core polarization effects included. The last data are available

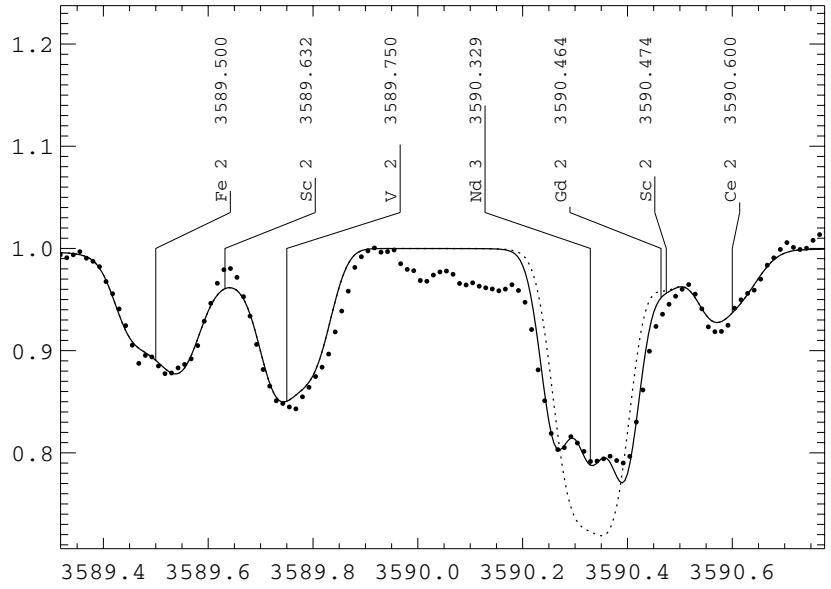

Fig. 5. The fit for Nd III lines to the observed HD 144897 spectrum (dots) in the $3590 \AA$ region. A dashed line represents previous classification for the Nd III $3590.24 \AA$ feature, while a solid line shows new classification for this line (see Sect. 5).

for the larger number of spectral lines and were used in our abundance analysis (Table 2). With only the experimental data we get $\log \left(\mathrm{Sm} / N_{\text {tot }}\right)=-7.03 \pm 0.23$ from 3 spectral lines. The $\mathrm{HF}$ calculations with the experimental BR give $\log \left(\mathrm{Sm} / N_{\text {tot }}\right)=$ $-6.90 \pm 0.14$ for 8 spectral lines. Abundance determinations with 3 sets of oscillator strengths agree within the error limits. Calculated Lande factors for the lines used in the abundance analysis represent the observed Zeeman patterns well.

Europium. Experimental oscillator strengths and $h f s$ constants for Eu II lines were taken from Lawler et al. (2001b). For the Eu III lines we again used the new transition probabilities and Landé factors calculations, which are based on extended analysis of the Eu III energy levels (Wyart et al. 2006). Generally, the new line parameters do not differ significantly from the DREAM data. $\mathrm{Eu}$ is the only rare-earth element for which more than a 1.0 dex difference between abundances from the lines of the first and second ions is obtained. The NLTE effects may explain about half of the observed difference in the case of homogeneous Eu distribution (see Mashonkina et al. 2002). NLTE effects of the same order are expected for Nd. Under the LTE 


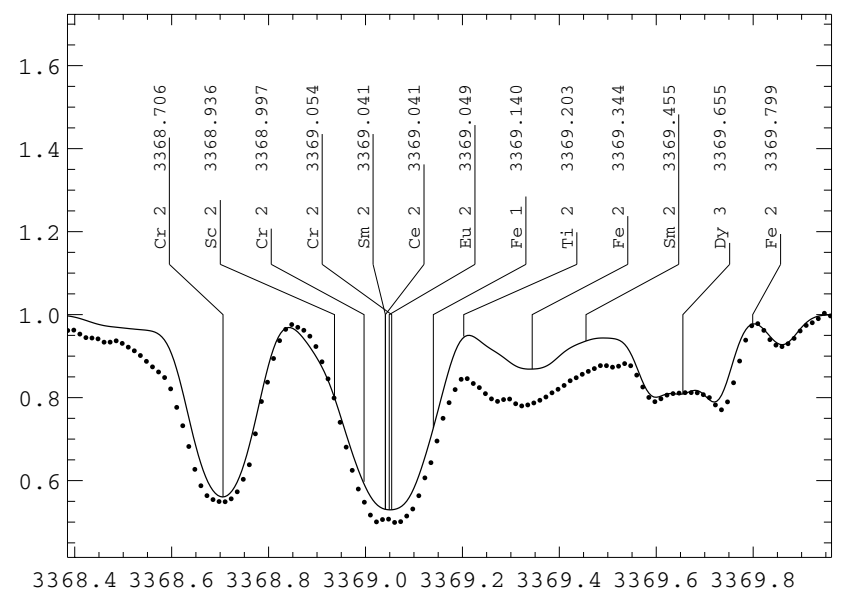

Fig. 6. The same as in Fig. 4 but for the Dy III line.

assumption, however, Nd II lines should provide lower abundances than Nd III lines (L. Mashonkina, private communication), but we do not find this discrepancy for HD 144897.

Gadolinium. The Gd III oscillator strengths are taken from Biémont et al. (2002b). Although partially blended, the two lines, $\lambda \lambda 3118.04$ and $3176.66 \AA$, are synthesized satisfactorily with the theoretical Landé factors.

Terbium. Experimental data for Tb II lines were adopted from Lawler et al. (2001c). For Tb III the transition probabilities and Landé factors were calculated by one of us (AR) based on the extended analysis of the Tb III energy levels (Wyart et al. 2006). Figure 4 gives an example of the fit to the observed Tb III 5847.23 A line. All other unblended lines in our analysis are also fitted perfectly with the current atomic data. The Tb III transition probabilities were also calculated by Biémont et al. (2002c), and the two sets of parameters agree within 0.05 dex.

Dysprosium. Experimental data for Dy II lines (Wickliffe et al. 2000) and theoretical calculations for Dy III lines (Zhang et al. 2002c) were used in the present study. Figure 6 shows the fit for one of the Dy III lines at $\lambda 3669.66 \AA$. Although a standard deviation in abundance obtained from Dy III lines is larger than for other species, good agreement between abundances derived from both ionization stages provides support for relatively accurate theoretical atomic parameters of Dy III lines.

Holmium. Experimental data for Ho II lines are taken from Lawler et al. (2004). Improved partition functions for Ho II (Bord \& Cowley 2002) were implemented in SYNTHMAG. The Ho III lines were synthesized using oscillator strengths calculated by Zhang et al. (2002a) and normalized to the measured lifetimes. Figure 7 demonstrates the fit of the calculated Ho III $3581.45 \AA$ line (the strongest in our study) to the observed spectrum. Obviously, $h f s$ effects are not significant. A fit of similar quality is obtained for other Ho III lines.

Erbium. For Er III calculations, we used the oscillator strength from Biémont et al. (2001a). Figure 8 illustrates the quality of existing Er III data.

Thulium. Experimental data for Tm II lines (Wickliffe \& Lawler 1997) and theoretical calculations for Tm III lines (Li et al. 2001) were used. The lines of both species are not strong. Nevertheless, a good agreement between the synthesized lines and the observed Zeeman splitting confirms reliability of the atomic data (see Fig. 9 for Tm III $3629.09 \AA$ ).

Ytterbium. Theoretical oscillator strengths (Biémont et al. 2001b) were used in the $\mathrm{Yb}$ III analysis. It was possible to model

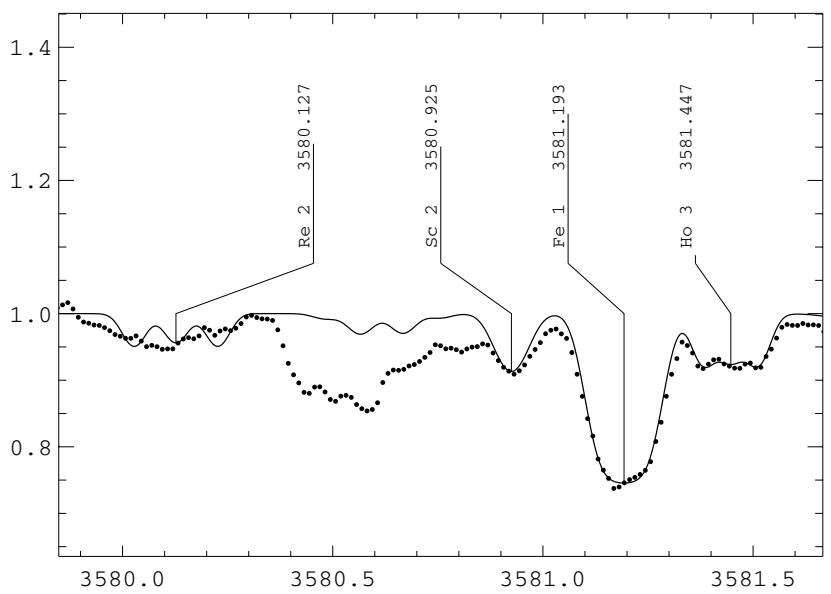

Fig. 7. The same as in Fig. 4 but for the Ho III line.

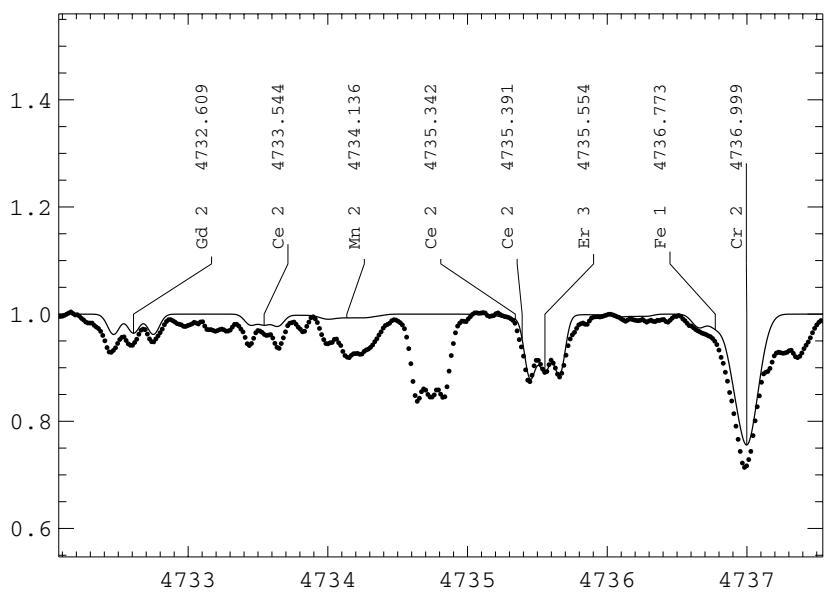

Fig. 8. The same as in Fig. 4 but for the Er III line.

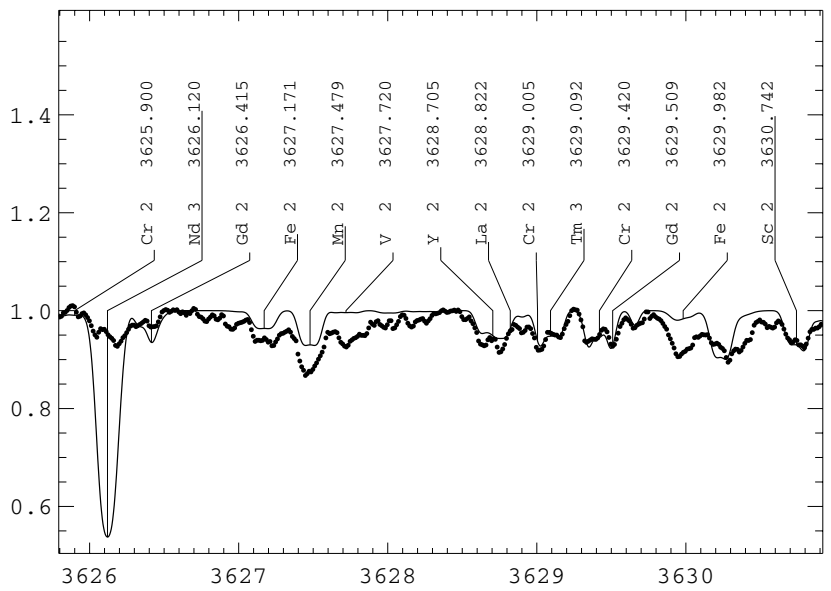

Fig. 9. The same as in Fig. 4 but for the Tm III line. No strong feature is observed at $3626.12 \AA$ corresponding to a strong Nd III line as predicted by Zhang et al. (2002b) on the basis of the Martin et al. (1978) energy levels.

only one line for each $\mathrm{Yb}$ ion. These lines are weak but provide consistent abundance.

Lutecium. The only line that is suitable for the Lu abundance determination is Lu II $6221.89 \AA$ A. It is weak, but in order to fit the line profile we have to account for both the Zeeman and $h f s$ splittings. At the same time, derived abundance is not affected by these effects. Oscillator strength for this line was taken 

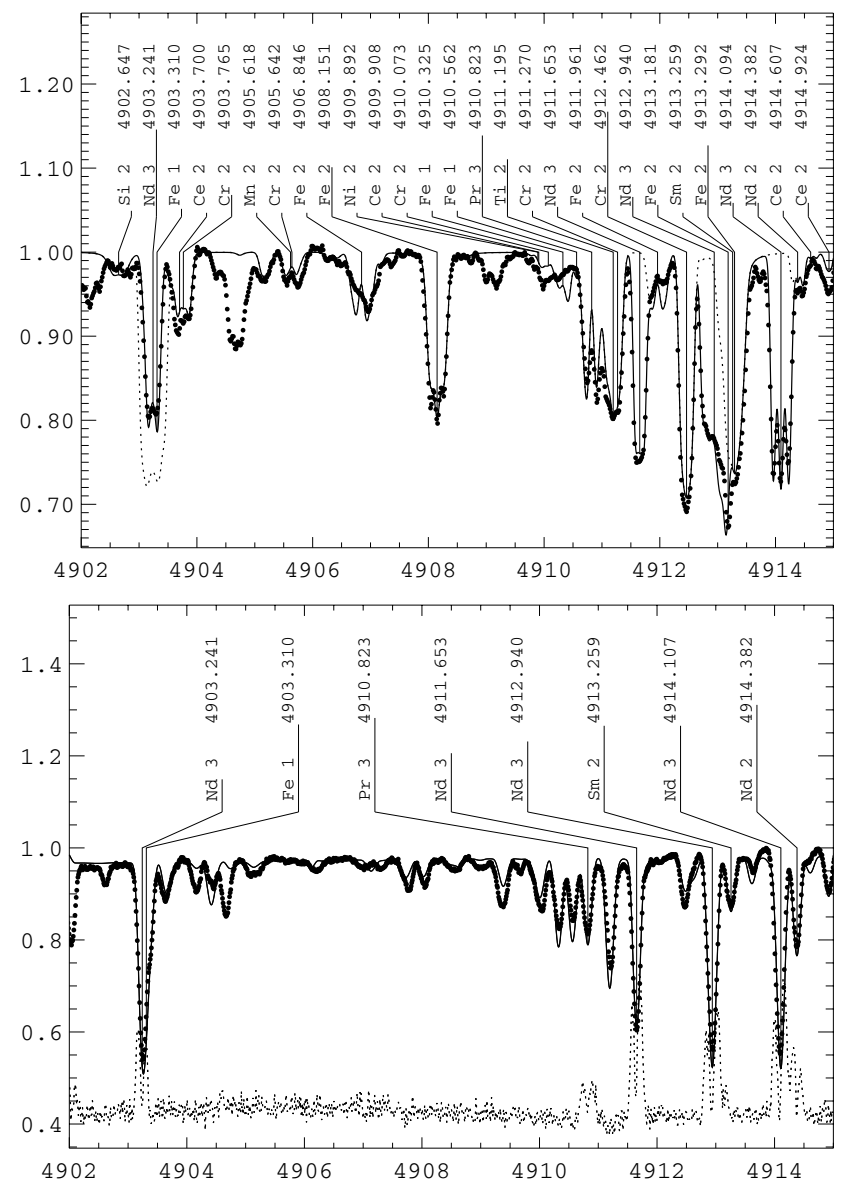

Fig. 10. The upper panel compares the observed spectrum of HD 144897 (dots) in the 4902-4915 A region with the spectrum synthesis based on the old (dotted line) and revised (solid line) classifications of the Nd III spectrum. The lower panel shows the same spectral interval for the roAp star HD 24712. The dotted curve gives standard deviation (arbitrary scale) as a function of wavelength for the time-resolved observations of HD 24712. Double-peaked bumps in the standard deviation indicate lines showing large-amplitude radial velocity variation.

from Den Hartog et al. (1998) and $h f s$ constants - from Brix \& Kopfermann (1952). Our synthesis confirmed the revised wavelength of this line, $\lambda 6221.86 \AA$, as derived by Bord et al. (1998) from the analysis of the solar spectrum.

Spectral synthesis of the REEs in HD 144897 provides strong support for the calculated atomic parameters for the second ions of most of these elements, hence, for the correct line classification. Only for Nd III, do we obtain inconsistencies between the theoretical calculations and a few rather strong observed features, which disagree either in the Zeeman pattern (Fig. 5) or in the intensity (Fig. 9). Taking into account that for all the second ions of REE calculations provide quite reliable atomic data, we may conclude that poor knowledge of the Nd III spectrum rather than a problem with the theoretical line profile calculations is the reason for the observed inconsistencies in the case of Nd III lines.

\section{Nd III classification}

The Nd III spectrum is very poorly known. Martin et al. (1978) compiled 5 levels of the $4 \mathrm{f}^{4}{ }^{5}$ I ground term and 24 levels of the $4 \mathrm{f}^{3} 5 \mathrm{~d}$ configuration using unpublished material by Crosswhite (1976). The latter list of Nd III lines was not published but circulates widely in the astrophysical community. Recent study of the Nd III spectrum was made by Aldenius (2001) by means of Fourier transform spectroscopy. She measured with a precision up to $0.005 \mathrm{~cm}^{-1}$ in wavenumbers the wavelengths of $58 \mathrm{Nd}$ III lines, 38 of which were identified with the levels published by Martin et al. (1978). The accuracy of the levels was significantly improved. It should be mentioned that Crosswite's and Aldenius' wavelength lists are partially inconsistent. Not all lines from Aldenius' list are present in Crosswhite's one, and some strong Crosswhite's lines are absent in Aldenius' list. Theoretical interpretation of the Nd III spectrum was performed by Bord (2000) and Zhang et al. (2002b). In the last paper the experimental lifetimes of five $4 \mathrm{f}^{3} 5 \mathrm{~d}$ levels were also reported.

Aldenius (2001) classified $38 \mathrm{Nd}$ III lines and suggested an identification of 20 new Nd III lines based on the laboratory spectrum, but did not provide their classification. In our classification, we used 27 the most intensive lines from her list of 38 classified lines and 9 lines from the list of unclassified ones. Rejected are the lines of low intensity (less than 3 in Aldenius' intensity scale) and the line $4781.014 \AA$ (intensity 10), which is practically invisible in the spectra of Ap and roAp stars. These spectra provide us with additional wavelength data. We mentioned in Sect. 1 that Nd III lines show outstanding pulsational characteristics in roAp stars, which we consider as a necessary (but not sufficient) condition for a spectral feature to belong to $\mathrm{Nd} \mathrm{III.}$ We chose a set of unidentified lines in the spectra of roAp stars $\gamma$ Equ (Kochukhov et al. 2004) and HD 24712 (Sachkov et al. 2006), which have pulsational amplitudes and phases similar to those for classified Nd III lines, and measured the wavelengths of these lines. Because of the large magnetic field, the spectrum of HD 144897 is not suitable for precise wavelength measurements; therefore, we carried out line-position determinations using the spectrum of roAp HD 217522. The moderate magnetic field $\left(\left\langle B_{\mathrm{s}}\right\rangle<2 \mathrm{kG}\right)$ and slow rotation $\left(v_{\mathrm{e}} \sin i \leq 3 \mathrm{~km} \mathrm{~s}^{-1}\right)$ of this star provide an accuracy $\sim 0.005 \AA$ for unblended lines. The spectrum of HD 217522 was obtained during the same observational program 68.D-0254 and was reduced in the same way as our data on HD 144897. Then we applied a classification procedure to the whole line sample using the code IDEN (Azarov 1993) for identifying the atomic spectra.

As in previous studies (Bord 2000; Zhang et al. 2002b), our calculations of the $\mathrm{Nd}$ III atomic structure were made with the Cowan code (Cowan 1981). To predict the effective energy parameters and electrostatic parameters in the $4 \mathrm{f}^{4}$ and $4 \mathrm{f}^{3}$ shells, we took advantage of the generalized parametric studies of doubly-ionized lanthanides (Wyart et al. 2006). Table 6 (Online material) lists the energy parameters of the $4 \mathrm{f}^{4}$ and $4 f^{3} 5 d$ configurations after fitting to all observed levels. The $4 f^{4}+4 f^{2} 5 d^{2}+4 f^{3} 6 p$ configurations were included in the even system of levels, while the $4 f^{3} 5 d+4 f^{3} 6 s+4 f^{2} 5 d 6 p$ configurations form the energy matrix of the odd set of levels. All electrostatic parameters for unknown configurations, as well as the parameters of configuration interactions, were fixed at the 0.85 level relative to the corresponding Hartree-Fock values. Average deviation $\sigma=55 \mathrm{~cm}^{-1}$ of our fitting is the same as in the previous calculations, but due to introduction of the effective energy parameters $\left(\alpha, \beta, \gamma\right.$ and $\left.\mathrm{F}^{1}(4 \mathrm{f}, 5 \mathrm{~d})\right)$ and scaling factors for $\mathrm{F}^{k}(4 \mathrm{f}, 4 \mathrm{f})$, the Slater integrals predictions for unknown energy levels are expected to be more accurate than in previous studies by Bord (2000) and Zhang et al. (2002b).

A list of identified Nd III lines is given in Table 4 and the energy levels found are presented in Table 7 (Online material). The first column of Table 4 contains recommended wavelengths for 71 lines. They coincide with the Aldenius' wavelengths where 

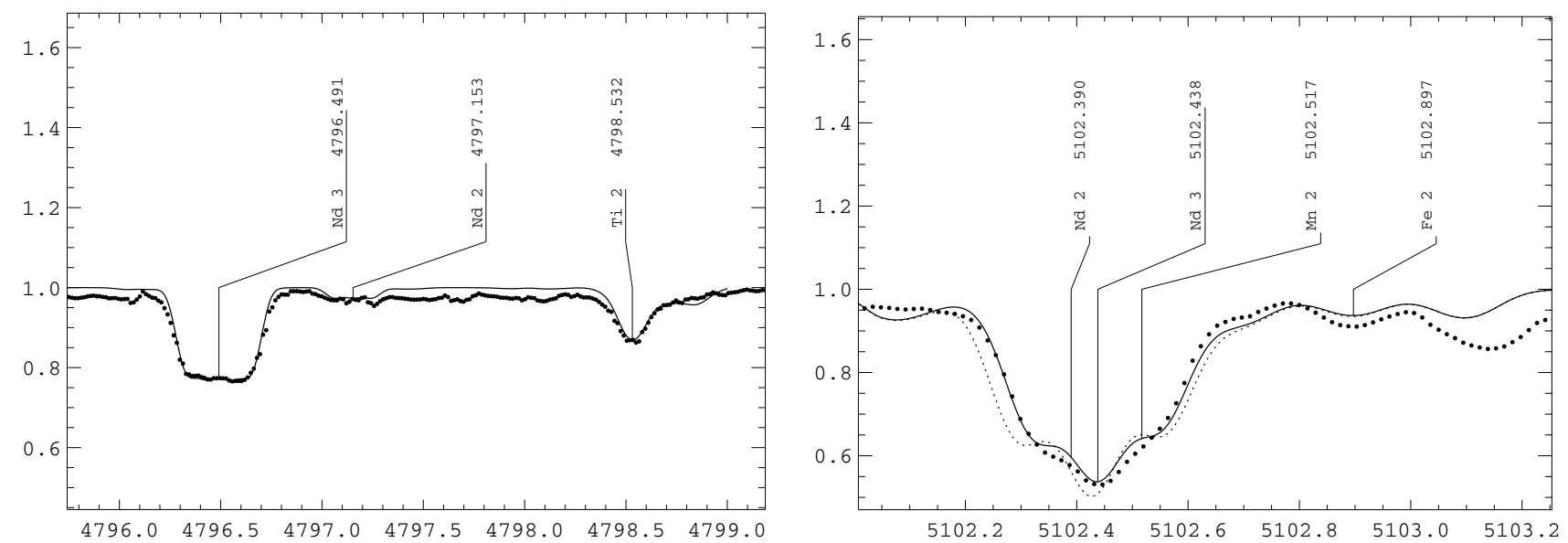

Fig. 11. The Nd III lines from the ${ }^{4} \mathrm{I}^{5} \mathrm{I}_{5,6}-{ }^{4} \mathrm{I}^{5} \mathrm{I}_{6}^{\mathrm{o}}$ transition array. Calculations with the old classification for $\lambda 5102.44$ line are shown by the dotted line.

available (Col. 2). Column 3 shows the wavelengths from the Crosswhite' list. In the 4th column the wavelengths measured in the spectrum of roAp star HD 217522 are given. For the lines absent in Aldenius' list but originating from the levels defined at least by one wavelength from Aldenius' list, we recommend the wavelengths calculated from the level energies (Ritz values). We also give Ritz values for transitions from some levels established by Crosswhite's wavelengths (where Aldenius' measurements were not available). In other cases, recommended wavelengths coincide with the stellar measurements.

An agreement between the theoretical Zeeman structure and intensity of lines from Table 4 (Online material) and the observed features in HD 144897 was accepted as a sufficient condition for correct Nd III classification. Mean abundance obtained for 39 newly classified lines is $\log \left(\mathrm{Nd} / N_{\text {tot }}\right)=-6.60 \pm 0.20$, which agrees very well with the abundance derived from the known lines (Table 2). Figure 10 gives an example of how newly classified Nd III lines fit the observed spectra in the 4900-4915 ̊ region. The top panel displays this spectral part in HD 144897, and the spectrum of the roAp star HD 24712 (Sachkov et al. 2006) is shown in the bottom panel with scaled pulsation pattern in roAp stars (standard deviation of the timeseries spectra from the averaged spectrum) where double peaks show position of the lines with pulsation radial velocity variations. Only one pulsating line, Nd III 4903.24 A, was previously known and classified, while another line 4914.09 $\AA$ was listed (but not classified) as Nd III by Aldenius (2001). Previous classification of the Nd III 4903.24 $\AA$ line does not correspond to the observed magnetic splitting (the upper panel of Fig. 10). Two other lines, 4911.65 and $4912.94 \AA$, with the same pulsation signature are also seen in Fig. 10. All 4 lines are classified in this work and the validity of the identification is proved by Zeeman calculations in the spectrum of HD 144897 (upper panel of Fig. 10). A good fit to the stellar spectrum for one more newly classified Nd III $5851.542 \AA$ line is shown in Fig. 4.

Table 7 (Online material) contains the energies of all levels of the $4 \mathrm{f}^{3} 5 \mathrm{~d}$ configuration with $J=3-10$ below $33000 \mathrm{~cm}^{-1}$. The energies of 35 levels were determined. We were able to confirm the energies only for 11 out of the 24 levels compiled by Martin et al. (1978). The oscillator strengths of 23 transitions from these 11 levels to the lower $4 \mathrm{f}^{4}{ }^{5} \mathrm{I}$ term, calculated by Zhang et al. (2002b) and in the present work, agree within 0.14 dex on average. The energies of almost all levels with $J=4-8$ are now based on the identification of 2 or 3 lines. The levels with $J=3$ are defined by one line and need further confirmation. Calculated composition of the levels (Col. 4) shows that some of the levels are highly mixed, and the leading contribution is given by the same LS term. To avoid possible ambiguities, we supplied designations of such levels by additional letters A or B in Table 4 (see also Table 7 of the online material).

Previous identification was changed for a few strong lines. For example, the changes were made for the $\left({ }^{4} \mathrm{I}\right)^{5} \mathrm{I}^{\mathrm{O}} J=5,6$, 8 levels, as follows.

$\left({ }^{4} \mathrm{I}\right)^{5} \mathrm{I}_{5}^{\mathrm{o}}$ : the new value $20348.715 \mathrm{~cm}^{-1}$ is supported by 3 lines, 5203.9236, 4912.946 and 5566.022 A, observed in stellar spectra with the intensities corresponding to the calculated $g f$ values. The previous energy of the level $20388.980 \mathrm{~cm}^{-1}$ was based on two lines, 5193.0397 and $4903.2410 \AA$, but the Zeeman structure calculated from this classification does not fit the observed spectrum of HD 144897. With the new classification these two lines match a transition array from the $\left({ }^{4} \mathrm{I}\right)^{5} \mathrm{H}_{4}^{\mathrm{o}}$ level perfectly (Fig. 10).

$\left({ }^{4} \mathrm{I}\right)^{5} \mathrm{I}_{6}^{\mathrm{o}}$ : Aldenius (2001) found only one line $4781.0141 \AA$ (mentioned above) as the ${ }^{5} \mathrm{I}_{5}-\left({ }^{4} \mathrm{I}\right)^{5} \mathrm{I}_{6}^{\mathrm{o}}$ transition to support the value $22048 \mathrm{~cm}^{-1}$ (Martin et al. 1978). Another expected transition to the ${ }^{5} \mathrm{I}_{6}^{\mathrm{o}}$ level at $5084.927 \AA$ with a 10 times larger transition probability was not measured by Aldenius. A rather strong line at $5085.0 \AA$ is present in the stellar spectra, but the predicted Zeeman pattern based on the Martin et al. energy levels does not fit the observed feature. There is no observable feature at $4781.014 \AA$ in any of our stars (HD 144897, $\gamma$ Equ, HD 24712, or HD 217522), which may be compared to a rather strong theoretically predicted line based on previous identification. Therefore, we reject previous energy for $\left({ }^{4} \mathrm{I}\right)^{5} \mathrm{I}_{6}^{\mathrm{O}}$ and propose instead two other lines 4796.4999 and 5102.4258 (blended by $\mathrm{Nd}$ II) for the transitions from the upper $\left({ }^{4} \mathrm{I}\right)^{5} \mathrm{I}_{6}^{\mathrm{O}}$ level to ${ }^{5} \mathrm{I}_{5,6}$ lower levels. Both lines are fitted fairly well with the new transition probabilities and Landé factors (Fig. 11). This identification is supported by the third weak line to the ${ }^{5} \mathrm{I}_{7}$ level at $5473.14 \AA$. The corrected $\left({ }^{4} \mathrm{I}\right)^{5} \mathrm{I}_{6}^{\mathrm{O}}$ energy for the level is $21980.505 \mathrm{~cm}^{-1}$.

$\left({ }^{4} \mathrm{I}\right)^{5} \mathrm{I}_{6}^{\mathrm{o}}$ : for previous value $24686.4 \mathrm{~cm}^{-1}$ (Martin et al. 1978) Aldenius (2001) found two lines at 5102.4278 and $4766.9971 \AA$. Theoretical calculations (Zhang et al. (2002b) predict a strong line at $4766.9971 \AA$, while no feature is observed in stellar spectra at this wavelength. We attributed the first line to the $\left({ }^{4} \mathrm{I}\right)^{5} \mathrm{I}_{6}^{\mathrm{O}}$ level (see above). The energy $24592.234 \mathrm{~cm}^{-1}$ of 
Table 3. Comparison between the measured and calculated lifetimes of the $5 \mathrm{~d}(4 \mathrm{~F})^{5} \mathrm{H}_{J}$ levels in a framework of new identifications.

\begin{tabular}{ccccc|cccc}
\hline \hline & \multicolumn{3}{c|}{ Zhang et al. (2002b) } & \multicolumn{3}{c}{ Present work } \\
& & \multicolumn{3}{c}{ Lifetime (ns) } & & \multicolumn{3}{c}{ Lifetime (ns) } \\
$J$ & $E\left(\mathrm{~cm}^{-1}\right)$ & $\lambda(\AA)$ & exp & theor & $E\left(\mathrm{~cm}^{-1}\right)$ & $\lambda(\AA)$ & exp & theor \\
\hline 3 & 27788.2 & 3598 & $110(10)$ & 110 & 27675.003 & 3612 & $70(5)$ & 87 \\
4 & 28745.3 & 3621 & $170(10)$ & 108 & 28745.285 & 3621 & $170(10)$ & 54 \\
5 & 30232.3 & 3590 & $105(15)$ & 108 & 30175.690 & 3598 & $110(10)$ & 69 \\
6 & 31394.6 & 3612 & $70(5)$ & 81 & 31559.191 & 3590 & $105(15)$ & 87 \\
7 & 32832.6 & 3604 & $87(7)$ & 98 & 32832.419 & 3604 & $87(7)$ & 86 \\
\hline
\end{tabular}

the $\left({ }^{4} \mathrm{I}\right)^{5} \mathrm{I}_{6}^{\mathrm{o}}$ level was derived based on the 5127.0441 and $4788.465 \AA$ lines.

Few relatively strong lines from the Aldenius' list remain unclassified, although for three of them (4514.128, 4711.333, $5028.520 \AA$ ) the Nd III identification is supported by pulsational observations in roAp stars.

\section{Discussion}

An extended abundance analysis of the magnetic Ap star HD 144897 strongly demonstrated a possibility of the adequate fit of the observed spectrum to the theoretical calculations when atmospheric (temperature, magnetic field, etc.) and atomic line parameters are correctly known. In particular, it concerns the rare-earth elements in the first and second ionization stages, which dominated the spectra of some cool roAp stars, for example, the famous Przybylski's star (HD 101065). Unfortunately, an extended laboratory analysis of some REEs in the second ionization stage is not complete. In such cases stellar data play an important role in spectrum classification. Usually, the quality of the theoretical atomic structure calculations are checked by comparing the observed and calculated lifetimes. For Nd III the lifetime measurements were made for 5 levels of the $5 \mathrm{~d}(4 \mathrm{~F})^{5} \mathrm{H}$ configuration having strong mixture with almost overlapping $5 \mathrm{~d}(4 \mathrm{~F})^{5} \mathrm{D}$ levels (Zhang et al. 2002b). The mixture results in the presence of two close lying levels with the $(4 \mathrm{~F})^{5} \mathrm{H}$ character, which were distinguished by additional letters A and B in our Table 4 (Online material). Because of the mixture, small variation of the energy parameters leads to drastic change of the calculated lifetimes.

A comparison between the observed and theoretical lifetimes calculated by Zhang et al. (2002b) and in the present work is given in Table 3. It should be mentioned that we suggested new energies for the $5 \mathrm{~d}(4 \mathrm{~F})^{5} \mathrm{H}$ levels with $J=3,5$, and 6 . Each level with $J=5$ or 6 is now based on two lines with the appropriate Zeeman structure checked using the HD 144897 spectrum. The $J=3$ level is defined by one relatively narrow line in accordance with the $g$-factor of this level. New classification corresponds better to the observed feature, although the calculated line is still narrower than the observed one. As a result we had to exchange the observed wavelengths and energies for $J=3,5$, and 6 levels according to new line classification.

One can notice an apparently better agreement between the measured and calculated lifetimes in Zhang et al. (2002b). However, there is a difficulty in the calculations of the oscillator strengths for the $5 \mathrm{p}^{6} 4 \mathrm{f}^{n}-5 \mathrm{p}^{6} 4 \mathrm{f}^{n-1} 5 \mathrm{~d}$ transitions related to the fact that the $4 \mathrm{f}$ electrons are deeply embedded inside the $5 \mathrm{p}^{5}$ core as discussed by Biémont et al. (2001c). As a result, the relativistic Hartree-Fock calculated lifetimes for the $4 \mathrm{f}^{n-1} 5 \mathrm{~d}$ levels are shorter than the measured ones. For example, in Eu III a ratio of measured and calculated lifetimes is equal to 3 (Mashonkina et al. 2002). In view of this property, the relation between calculated and measured lifetimes has an expected tendency in the present work, but not in the Zhang et al. (2002b) study.

\section{Conclusions}

We have presented an extensive abundance analysis, with the emphasis on REEs, in the atmosphere of the tepid magnetic Ap star HD 144897. Thanks to the extremely wide wavelength coverage of our observational material, all stable REEs but $\mathrm{La}$ and $\mathrm{Lu}$ are studied using spectral lines of the first and second ionization stages, thus giving more confidence to the REE abundance results. The spectrum of HD 144897 is very complex because of the large overabundance of the Fe-peak elements and Zeeman splitting of lines in a $8.8 \mathrm{kG}$ magnetic field. Therefore, a careful abundance and, in particular, stratification analysis of the most prominent elements, like $\mathrm{Cr}$ and $\mathrm{Fe}$, was performed to minimize blending problems in the REE analysis. Comparison of the atmospheric abundances in HD 144897 and in the other two tepid Ap stars, HD 170973 (Kato 2003) and HD 116458 (Nishimura et al. 2004), shows close similarity in the REE abundances, in particular. On average, REEs are overabundant by 4 dex compared to the solar abundances, and no serious violation of the odd-even effect is observed. Using a spectrum of the star where clearly resolved Zeeman patterns for most spectral lines are observed, we were able to check the accuracy of the experimental (first REE ions) and theoretical (second REE ions) data: energy level classification, transition probabilities, and Landé factors. We found that for all ions but Nd III, the profiles of unblended or partially blended REE lines in the spectrum of HD 144897 are well synthesized when adopting the value $8.8 \mathrm{kG}$ for the mean magnetic field modulus. In contrast, less than half of the previously classified Nd III lines could be fitted. Using two criteria for the Nd III line identification - peculiar pulsational characteristics in rapidly oscillating stars and a correspondence between the calculated and observed Zeeman pattern in magnetic star HD 144897 - we performed a revision of Nd III classification. We were able to confirm the energies only for 11 out of the 24 levels compiled by Martin et al. (1978), and we derived energies for additional 24 levels of the $4 f^{3} 5 d$ configuration increasing a total number of classified $\mathrm{Nd}$ III lines with corrected wavelengths and atomic parameters.

Our classification of the Nd III energy levels was partially based on stellar measurements. Therefore, precise laboratory measurements in wide spectral region are required to confirm our results.

Acknowledgements. We thank Prof. C.R. Cowley for providing us with the unpublished Nd III line lists by H. Crosswhite. TR acknowledges financial support from the RFBR grant 04-02-16788a, the Presidium of RAS Program "Origin, and Evolution of Stars and Galaxies" and the Austrian Science Fund (FWF-P17580N2). The project was partially supported by the grant 11630102 from the Royal Swedish Academy of Sciences. 


\section{References}

Aldenius, M. 2001, Master Thesis, Depart. of Physics, Univ. of Lund Asplund, M., Grevesse, N., \& Sauval, A. J. 2005, ASP Conf. Ser., 336, 25 Azarov, V. I. 1993, Phys. Scripta, 48, 656

Ballester, P., Modigliani, A., Boitquin, O., et al. 2000, Messenger, 101, 31 Biémont, E., Palmeri, P., \& Quinet, P. 1999, Ap\&SS, 635, 269

Biémont, E., Garnir, H. P., Bastin, T., et al. 2001a, MNRAS, 321, 482

Biémont, E., Garnir, H. P., Li, Z. S., et al. 2001b, J. Phys. B, 34, 1869

Biémont, E., Garnir, H. P., Palmeri, P., et al. 2001c, Phys. Rev., 64, 022503

Biémont, E., Quinet, P., \& Ryabchikova, T. A. 2002a, MNRAS, 336, 1155

Biémont, E., Kohnen, G., \& Quinet, P. 2002b, A\&A, 393, 717

Biémont, E., Garnir, H. P., Quinet, P., Svanberg, S., \& Zhang, Z. G. 2002c, Phys. Rev. A, 65, 052502

Biémont, E., Garnir, H. P., Litzén, U., et al. 2003, A\&A, 399, 343

Bord, D. J. 2000, A\&AS, 144, 517

Bord, D. J., \& Cowley, C. R. 2002, Solar Phys., 211, 3

Bord, D. J., Cowley, C. R., \& Mirijanian, D. 1998, Solar Phys., 178, 221

Brix, P., \& Kopfermann, H. 1952, in Landolt-Börnstein, Zahlenwerte und Funktionen, 6th ed. Vol.I, part 5 (Berlin: Springer-Verlag)

Burki, G., et al. 2005, GENEVA photometric database, Geneva Observatory

Cowan, R. D. 1981, The Theory of Atomic Structure and Spectra (Berkeley, CA, USA: Univ. California Press)

Crosswhite, H. 1976, unpublished data

Cramer, N., \& Maeder, A. 1980, A\&A, 88,135

Dekker, H., D'Odorico, S., Kaufer, A., Delabre, B., \& Kotzlowski, H. 2000, Proc. SPIE, 4008, 534

Den Hartog, E. A., Curry, J. J., Wickliffe, M. E., \& Lawler, J. E. 1998, Solar Phys., 178, 239

Den Hartog, E. A., Lawler, J. E., Sneden, C., \& Cowan, J. J. 2003, ApJS, 148, 543

Dolk, L., Wahlgren, G. M., Lundberg, H., Li, et al. 2002, A\&A, 385, 111

Ginibre, A. 1989, Phys. Scr., 39, 694

Glagolevskij, Yu. V., Ryabchikova, T. A., \& Chuntonov, G. A. 2005, Astron. Lett., 31,327

Hakkila, J., Myers, J. M., Stidham, B. J., \& Hartmann, D. H. 1997, AJ, 114, 2043

Hauck, B., \& Künzli, M. 1996, Baltic Astron., 5, 303

Holt, R. A., Scholl, T. J., \& Rosner, S. D. 1999, MNRAS, 306, 107

Kato, K. 2003, PASJ, 55, 1133

Kling, R., \& Griesmann, U. 2000, ApJ, 531, 1173

Kling, R., Schnabel, R., \& Griesmann, U. 2001, ApJS, 134, 173

Kochukhov, O., \& Ryabchikova, T. 2001a, A\&A, 374, 615

Kochukhov, O., \& Ryabchikova, T. 2001b, A\&A, 377, L22

Kochukhov, O., Ryabchikova, T., \& Piskunov, N. E. 2004, A\&A, 415, L13

Kochukhov, O., Tsymbal, V., Ryabchikova, T., Makaganyk, V., \& Bagnulo, S. 2006 , in preparation

Künzli, M., North, P., Kurucz, R. L., \& Nicolet, B. 1997, A\&AS, 122, 51

Kupka, F., Piskunov, N., Ryabchikova, T. A., Stempels, H. C., \& Weiss, W. W. 1999, A\&AS, 138, 119
Kurucz, R. L. 1993, Kurucz CD-ROM 13, Cambridge, SAO

Lawler, J. E., Bonvallet, G., \& Sneden, C. 2001a, ApJ, 556, 452

Lawler, J. E., Wickliffe, M. E., Den Hartog, E. A., \& Sneden, C. 2001b, ApJ, 563,1075

Lawler, J. E., Wickliffe, M. E., Cowley, C. R., \& Sneden, C. 2001c, ApJS, 137, 341

Lawler, J. E., Sneden, C., \& Cowan, J. J. 2004, ApJ, 604, 850

Lawler, J. E., Den Hartog, E. A., Sneden, C., \& Cowan, J. J. 2006, ApJS, 162, 227

Li, Z. S., Zhang, Z. G., Lokhnygin, V., et al. 2001, J. Phys. B, 34, 1369

Lucke, P. B. 1978, A\&A, 64, 367

Martin, W. C., Zalubas, R., \& Hagan, L. 1978, NSRDS-NBS 60, Washington

Mashonkina, L. I., Ryabtsev, A. N., \& Ryabchikova, T. A. 2002, Astron. Lett., 28,34

Mashonkina, L. I., Ryabchikova, T. A., \& Ryabtsev, A. N. 2005, A\&A, 441, 309

Mathys, G., Hubrig, S., Landstreet, J. D., Lanz, T., \& Manfroid, J. 1997, A\&AS, 123,353

Moore, C. E. 1971, NSRDS-NBS 35, volume II, III

Munari, U., \& Zwitter, T. 1997, A\&A, 318, 269

Nishimura, M., Sadakane, K., Kato, K., Takeda, Y, \& Mathys, G. 2004, A\&A, 420,673

Palmeri, P., Quinet, P., Wyart, J.-F., \& Biémont, E. 2000, Phys. Scr., 61, 323

Perryman, M. A. C., Lindegren, L., Kovalevsky, J., et al. 1997, A\&AS, 323, 49

Pickering, J. C., Thorne, A. P., \& Perez, R. 2001, ApJS, 132, 403

Piskunov, N. E. 1999, in 2nd International Workshop on Solar Polarization, ed. K. Nagendra, \& J. Stenflo (Kluwer Acad. Publ.), ASSL, 243, 515

Quinet, P., \& Biémont, E. 2004, At. Data Nucl. Data Tables, 87, 207

Raassen, A. J. J., \& Uylings, P. H. M. 1998, A\&A, 340, 300

Ryabchikova, T., Leone, F., \& Kochukhov, O. 2005, A\&A, 438, 973

Ryabchikova, T. A., Savanov, I. S., Hatzes, A. P., Weiss, W. W., \& Handler, G. 2000, A\&A, 357, 981

Sachkov, M., Ryabchikova, T., Bagnulo, S., et al. 2006, in Stellar Pulsation and Evolution, ed. A. R. Walker, \& G. Bono, Mem. Soc. Astron. It., 77, 397 [arXiv: astro-ph/0509748]

Savanov, I. S., Malanushenko, V. P., \& Ryabchikova, T. A. 1999, AstL, 25, 802

Schlegel, D. J., Finkbeiner, D. P., \& Davis, M. 1998, ApJ, 500, 525

Shulyak, D., Tsymbal, V., Ryabchikova, T., Stütz, Ch, \& Weiss, W. W. 2004, A\&A, 428, 993

Swings, P. 1944, ApJ, 100, 132

Wickliffe, M. E., \& Lawler, J. E. 1997, JOSA, B14, 737

Wickliffe, M. E., Lawler, J. E., \& Nave, G. 2000, JQSRT, 66, 363

Wilke, R. 2003, Ph.D. Thesis, Heinrich-Heine-Universität, Düsseldorf

Wyart, J.-F., Tchang-Brillet, W.-Ü.L., Churilov, S. S., \& Ryabtsev, A. N. 2006, Phys. Scr., in press

Zhang, Z. G., Somesfalean, G., Svanberg, S., et al. 2002a, A\&A, 384, 364

Zhang, Z. G., Svanberg, S., Palmeri, P., Quinet, P., \& Biémont, E. 2002b, A\&A, 385,724

Zhang, Z. G., Svanberg, S., Palmeri, P., Quinet, P., \& Biémont, E. 2002c, MNRAS, 334, 1 
T. Ryabchikova et al.: Rare-earth elements in the atmosphere of the magnetic chemically peculiar star HD 144897, Online Material p 1

\section{Online Material}


T. Ryabchikova et al.: Rare-earth elements in the atmosphere of the magnetic chemically peculiar star HD 144897, Online Material p 2

Table 4. Proposed classification for Nd III lines.

\begin{tabular}{|c|c|c|c|c|c|c|c|c|c|c|c|}
\hline \multicolumn{4}{|c|}{ Wavelength $(\AA)$} & \multicolumn{4}{|c|}{ Lower level $^{a}$} & \multicolumn{4}{|c|}{$\overline{\text { Upper level }}$} \\
\hline This work $^{b}$ & $\exp ^{c}$ & $\exp ^{d}$ & Stellar ${ }^{e}$ & $\log (g f)$ & $E\left(\mathrm{~cm}^{-1}\right)$ & $J$ & $g$ & $E\left(\mathrm{~cm}^{-1}\right)$ & $J$ & $g$ & LS term $^{f}$ \\
\hline $3427.0017 \mathrm{R}$ & & & $.00 \mathrm{bl}$ & -1.69 & 2387.529 & 6.0 & 1.071 & 31559.191 & 6.0 & 1.151 & $\left({ }^{4} \mathrm{~F}\right)^{5} \mathrm{H}^{0} \mathrm{~B}$ \\
\hline 3433.3315 & .3315 & .327 & $.33 \mathrm{bl}$ & -1.70 & 3714.537 & 7.0 & 1.177 & 32832.420 & 7.0 & 1.155 & $\left({ }^{4} \mathrm{~F}\right)^{5} \mathrm{H}^{\mathrm{o}} \mathrm{B}$ \\
\hline 3442.7891R & & .781 & .793 & -1.49 & 1137.795 & 5.0 & 0.902 & 30175.690 & 5.0 & 1.051 & $\left({ }^{4} \mathrm{~F}\right)^{5} \mathrm{H}^{0} \mathrm{~B}$ \\
\hline $3476.1892 \mathrm{R}$ & & .184 & .192 & -2.00 & 2387.529 & 6.0 & 1.071 & 31146.429 & 6.0 & 1.060 & $\left({ }^{4} \mathrm{~F}\right)^{5} \mathrm{H}^{\mathrm{o}} \mathrm{A}$ \\
\hline 3477.8359 & .8359 & .834 & .839 & -1.66 & 0.000 & 4.0 & 0.605 & 28745.285 & 4.0 & 0.916 & $\left({ }^{4} \mathrm{~F}\right)^{5} \mathrm{H}^{\mathrm{o}}$ \\
\hline 3537.6116 & .6116 & .607 & $.63 \mathrm{bl}$ & -2.60 & 1137.795 & 5.0 & 0.902 & 29397.378 & 5.0 & 0.982 & $\left({ }^{4} \mathrm{~F}\right)^{5} \mathrm{H}^{\mathrm{o}} \mathrm{A}$ \\
\hline 3561.8555R & & & .858 & -2.31 & 3714.537 & 7.0 & 1.177 & 31781.776 & 7.0 & 1.141 & $\left({ }^{4} \mathrm{~F}\right)^{5} \mathrm{H}^{\mathrm{o}} \mathrm{A}$ \\
\hline 3590.3291 & .3291 & .332 & .330 & -0.59 & 3714.537 & 7.0 & 1.177 & 31559.191 & 6.0 & 1.151 & $\left({ }^{4} \mathrm{~F}\right)^{5} \mathrm{H}^{\mathrm{o}} \mathrm{B}$ \\
\hline 3597.6284 & .6284 & .629 & .630 & -0.57 & 2387.529 & 6.0 & 1.071 & 30175.690 & 5.0 & 1.051 & $\left({ }^{4} \mathrm{~F}\right)^{5} \mathrm{H}^{0} \mathrm{~B}$ \\
\hline 3603.9826 & .9826 & & .982 & -0.55 & 5093.250 & 8.0 & 1.247 & 32832.420 & 7.0 & 1.155 & $\left({ }^{4} \mathrm{~F}\right)^{5} \mathrm{H}^{\circ} \mathrm{B}$ \\
\hline 3612.3388 & .3388 & & .338 & -0.80 & 0.000 & 4.0 & 0.605 & 27675.003 & 3.0 & 0.836 & $\left({ }^{4} \mathrm{~F}\right)^{5} \mathrm{H}^{\mathrm{o}}$ \\
\hline 3621.1727 & .1727 & .171 & $.18 \mathrm{bl}$ & -0.52 & 1137.795 & 5.0 & 0.902 & 28745.285 & 4.0 & 0.916 & $\left({ }^{4} \mathrm{~F}\right)^{5} \mathrm{H}^{\mathrm{o}}$ \\
\hline 3644.3534 & .3534 & & .357 & -0.85 & 3714.537 & 7.0 & 1.177 & 31146.429 & 6.0 & 1.060 & $\left({ }^{4} \mathrm{~F}\right)^{5} \mathrm{H}^{\mathrm{o}} \mathrm{A}$ \\
\hline 3701.3000 & .3000 & .288 & .32 & -1.06 & 2387.529 & 6.0 & 1.071 & 29397.378 & 5.0 & 0.982 & $\left({ }^{4} \mathrm{~F}\right)^{5} \mathrm{H}^{\mathrm{o}} \mathrm{A}$ \\
\hline 3745.8637 & .8637 & & .86 & -0.62 & 5093.250 & 8.0 & 1.247 & 31781.776 & 7.0 & 1.141 & $\left({ }^{4} \mathrm{~F}\right)^{5} \mathrm{H}^{\mathrm{o}} \mathrm{A}$ \\
\hline 4211.003 & & .003 & .014 & -2.43 & 1137.795 & 5.0 & 0.902 & 24878.420 & 5.0 & 1.141 & $\left({ }^{4} \mathrm{I}^{3} \mathrm{H}^{\mathrm{o}}\right.$ \\
\hline $4213.3602 R$ & & .356 & .367 & -2.66 & 3714.537 & 7.0 & 1.177 & 27441.879 & 8.0 & 1.140 & $\left({ }^{4} \mathrm{I}\right)^{3} \mathrm{~K}^{\mathrm{O}}$ \\
\hline $4359.485 \mathrm{R}$ & & & $.48 \mathrm{bl}$ & -3.03 & 2387.529 & 6.0 & 1.071 & 25319.572 & 7.0 & 1.099 & $\left({ }^{4} \mathrm{I}\right)^{3} \mathrm{~K}^{\mathrm{O}}$ \\
\hline 4414.300 & & .300 & .312 & -2.22 & 2387.529 & 6.0 & 1.071 & 25034.830 & 6.0 & 1.250 & $\left({ }^{4} I\right)^{5} \mathrm{G}^{0}$ \\
\hline 4444.997 R & & & 5.01 & -2.22 & 2387.529 & 6.0 & 1.071 & 24878.420 & 5.0 & 1.141 & $\left({ }^{4} \mathrm{I}\right)^{3} \mathrm{H}^{\mathrm{o}}$ \\
\hline 4466.355 & & & .355 & -2.52 & 3714.537 & 7.0 & 1.177 & 26097.872 & 7.0 & 1.186 & $\left({ }^{4} \mathrm{I}\right)^{5} \mathrm{H}^{\mathrm{o}}$ \\
\hline 4473.2922 & .2922 & .287 & .292 & -1.30 & 5093.250 & 8.0 & 1.247 & 27441.879 & 8.0 & 1.140 & $\left({ }^{4} \mathrm{I}\right)^{3} \mathrm{~K}^{\mathrm{o}}$ \\
\hline 4501.2346R & & & blend & -2.03 & 1137.795 & 5.0 & 0.902 & 23347.692 & 5.0 & 1.153 & $\left({ }^{4} I\right)^{5} \mathrm{G}^{\mathrm{o}}$ \\
\hline 4507.0450R & & & $.06 \mathrm{bl}$ & -3.24 & 0.000 & 4.0 & 0.605 & 22181.265 & 5.0 & 1.032 & $\left({ }^{4} \mathrm{I}\right)^{5} \mathrm{H}^{\mathrm{o}}$ \\
\hline 4570.660 & & .660 & .643 & -2.06 & 1137.795 & 5.0 & 0.902 & 23010.344 & 4.0 & 1.031 & $\left({ }^{4} I\right)^{5} \mathrm{G}^{0}$ \\
\hline 4613.1780R & & & .211 & -3.14 & 2387.529 & 6.0 & 1.071 & 24058.489 & 6.0 & 1.122 & $\left({ }^{4} \mathrm{I}\right)^{5} \mathrm{H}^{\mathrm{O}}$ \\
\hline 4624.9799 & .9799 & .965 & $.97 \mathrm{bl}$ & -1.88 & 2387.529 & 6.0 & 1.071 & 24003.190 & 7.0 & 1.176 & $\left({ }^{4} \mathrm{I}\right)^{5} \mathrm{I}^{0} \mathrm{~B}$ \\
\hline 4627.254 & & .254 & .254 & -2.08 & 3714.537 & 7.0 & 1.177 & 25319.572 & 7.0 & 1.099 & $\left({ }^{4} \mathrm{I}\right)^{3} \mathrm{~K}^{\mathrm{o}}$ \\
\hline 4651.6225 & .6225 & .609 & .617 & -1.86 & 0.000 & 4.0 & 0.605 & 21491.858 & 4.0 & 0.948 & $\left({ }^{4} \mathrm{I}^{3} \mathrm{H}^{\mathrm{o}}\right.$ \\
\hline 4654.312 & & .312 & .310 & -1.92 & 0.000 & 4.0 & 0.605 & 21479.437 & 3.0 & 0.825 & $\left({ }^{4} I\right)^{5} \mathrm{G}^{0}$ \\
\hline 4689.052 & & .052 & .055 & -1.80 & 3714.537 & 7.0 & 1.177 & 25034.830 & 6.0 & 1.250 & $\left({ }^{4} I\right)^{5} \mathrm{G}^{\mathrm{o}}$ \\
\hline 4759.526 & & .499 & .526 & -1.15 & 5093.250 & 8.0 & 1.247 & 26097.872 & 7.0 & 1.186 & $\left({ }^{4} \mathrm{I}^{5} \mathrm{H}^{\mathrm{O}}\right.$ \\
\hline 4769.6217 & .6217 & .610 & .610 & -1.80 & 2387.529 & 6.0 & 1.071 & 23347.692 & 5.0 & 1.153 & $\left({ }^{4} I\right)^{5} \mathrm{G}^{0}$ \\
\hline 4788.4617R & & .423 & .465 & -1.77 & 3714.537 & 7.0 & 1.177 & 24592.234 & 8.0 & 1.229 & $\left({ }^{4} \mathrm{I}\right)^{5} \mathrm{I}^{\mathrm{O}}$ \\
\hline 4796.4999 & .4999 & .465 & .499 & -1.65 & 1137.795 & 5.0 & 0.902 & 21980.505 & 6.0 & 1.084 & $\left({ }^{4} I\right)^{5} I^{0}$ \\
\hline 4821.986 & & .986 & .988 & -2.45 & 2387.529 & 6.0 & 1.071 & 23120.081 & 6.0 & 0.940 & $\left({ }^{4} \mathrm{I}\right)^{3} \mathrm{~K}^{\mathrm{o}}$ \\
\hline 4903.2410 & .2410 & & $.26 \mathrm{bl}$ & -2.21 & 0.000 & 4.0 & 0.605 & 20388.980 & 4.0 & 0.836 & $\left({ }^{4} \mathrm{I}\right)^{5} \mathrm{H}^{\mathrm{o}}$ \\
\hline 4911.6527R & & & .658 & -1.63 & 1137.795 & 5.0 & 0.902 & 21491.858 & 4.0 & 0.948 & $\left({ }^{4} \mathrm{I}\right)^{3} \mathrm{H}^{\mathrm{o}}$ \\
\hline 4912.9436R & & & .946 & -1.78 & 0.000 & 4.0 & 0.605 & 20348.715 & 5.0 & 0.924 & $\left({ }^{4} \mathrm{I}\right)^{5} \mathrm{I}^{\mathrm{O}}$ \\
\hline 4914.0941 & .0941 & & .095 & -1.10 & 3714.537 & 7.0 & 1.177 & 24058.489 & 6.0 & 1.122 & $\left({ }^{4} \mathrm{I}^{5} \mathrm{H}^{\mathrm{o}}\right.$ \\
\hline 4921.0431 & .0431 & & $.04 \mathrm{bl}$ & -1.89 & 2387.529 & 6.0 & 1.071 & 22702.749 & 7.0 & 1.146 & $\left({ }^{4} \mathrm{I}\right)^{5} \mathrm{I}^{\mathrm{o}} \mathrm{A}$ \\
\hline 4927.4877 & .4877 & .484 & .484 & -0.80 & 3714.537 & 7.0 & 1.177 & 24003.190 & 7.0 & 1.176 & $\left({ }^{4} \mathrm{I}\right)^{5} \mathrm{I}^{\mathrm{o}} \mathrm{B}$ \\
\hline 4942.673 & & .673 & 6 & -1.23 & 5093.250 & 8.0 & 1.247 & 25319.572 & 7.0 & 1.099 & $\left({ }^{4} \mathrm{I}\right)^{3} \mathrm{~K}^{\mathrm{o}}$ \\
\hline 5050.6952 & .6952 & & .700 & -1.06 & 2387.529 & 6.0 & 1.071 & 22181.265 & 5.0 & 1.032 & $\left({ }^{4} \mathrm{I}\right)^{5} \mathrm{H}^{\mathrm{o}}$ \\
\hline 5084.6597R & & & .660 & -2.58 & 1137.795 & 5.0 & 0.902 & 20799.314 & 6.0 & 1.021 & $\left({ }^{4} \mathrm{I}\right)^{3} \mathrm{I}^{\mathrm{O}}$ \\
\hline 5102.4278 & .4278 & .430 & .437 & -0.62 & 2387.529 & 6.0 & 1.071 & 21980.505 & 6.0 & 1.084 & $\left({ }^{4} \mathrm{I}\right)^{5} \mathrm{I}^{\mathrm{O}}$ \\
\hline 5127.0441 & .0441 & .009 & .049 & -0.40 & 5093.250 & 8.0 & 1.247 & & 8.0 & 1.229 & $\left({ }^{4} \mathrm{I}\right)^{5} \mathrm{I}^{\mathrm{O}}$ \\
\hline $5151.731 \mathrm{R}$ & & & .742 & -1.54 & 3714.537 & 7.0 & 1.177 & 81 & 6.0 & 0.940 & $\left({ }^{4} \mathrm{I}\right)^{3} \mathrm{~K}^{\mathrm{o}}$ \\
\hline 5152.292 & & .292 & $.25 \mathrm{bl}$ & -1.21 & 0.000 & 4.0 & 0.605 & 19403.433 & 3.0 & 0.575 & $\left({ }^{4} \mathrm{I}\right)^{5} \mathrm{H}^{\mathrm{o}}$ \\
\hline 5193.0397 & .0397 & .061 & .038 & -1.18 & 1137.795 & 5.0 & 0.902 & 20388.980 & 4.0 & 0.836 & $\left({ }^{4} \mathrm{I}\right)^{5} \mathrm{H}^{\mathrm{o}}$ \\
\hline 5203.9236 & .9236 & .906 & .922 & -0.66 & 1137.795 & 5.0 & 0.902 & 20348.715 & 5.0 & 0.924 & $\left({ }^{4} \mathrm{I}\right)^{5} \mathrm{I}^{\mathrm{O}}$ \\
\hline 5264.9604 & .9604 & .959 & .965 & -0.72 & 3714.537 & 7.0 & 1.177 & 22702.749 & 7.0 & 1.146 & $\left({ }^{4} \mathrm{I}\right)^{5} \mathrm{I}^{\mathrm{o}} \mathrm{A}$ \\
\hline 5286.7534 & .7534 & & .728 & -1.90 & 5093.250 & 8.0 & 1.247 & 24003.190 & 7.0 & 1.176 & $\left({ }^{4} \mathrm{I}\right)^{5} \mathrm{I}^{0} \mathrm{~B}$ \\
\hline 5294.1133 & .1133 & .106 & .108 & -0.69 & 0.000 & 4.0 & 0.605 & 18883.652 & 4.0 & 0.640 & $\left({ }^{4} \mathrm{I}\right)^{5} \mathrm{I}^{\mathrm{O}}$ \\
\hline 5410.0994 & .0994 & & .098 & -1.52 & 1137.795 & 5.0 & 0.902 & 19616.608 & 5.0 & 0.869 & $\left({ }^{4} \mathrm{I}\right)^{3} \mathrm{I}^{\mathrm{O}}$ \\
\hline 5429.7944 & .7944 & & .793 & -1.24 & 2387.529 & 6.0 & 1.071 & 20799.314 & 6.0 & 1.021 & $\left({ }^{4} \mathrm{I}\right)^{3} \mathrm{I}^{\mathrm{O}}$ \\
\hline $5473.1411 \mathrm{R}$ & & & $.14 \mathrm{bl}$ & -3.03 & 3714.537 & 7.0 & 1.177 & 21980.505 & 6.0 & 1.084 & $\left({ }^{4} \mathrm{I}\right)^{5} \mathrm{I}^{\mathrm{O}}$ \\
\hline $5566.0154 \mathrm{R}$ & & & .022 & -2.33 & 2387.529 & 6.0 & 1.071 & 20348.715 & 5.0 & 0.924 & $\left({ }^{4} \mathrm{I}\right)^{5} \mathrm{I}^{\mathrm{O}}$ \\
\hline 5633.5540 & .5540 & & .558 & -2.22 & 1137.795 & 5.0 & 0.902 & 18883.652 & 4.0 & 0.640 & $\left({ }^{4} \mathrm{I}\right)^{5} \mathrm{I}^{\mathrm{O}}$ \\
\hline 5677.1788 & .1788 & & .189 & -1.45 & 5093.250 & 8.0 & 1.247 & 22702.749 & 7.0 & 1.146 & $\left({ }^{4} \mathrm{I}\right)^{5} \mathrm{I}^{\mathrm{o}} \mathrm{A}$ \\
\hline 5802.5319R & & & .536 & -1.71 & 2387.529 & 6.0 & 1.071 & 19616.608 & 5.0 & 0.869 & $\left({ }^{4} \mathrm{I}\right)^{3} \mathrm{I}^{\mathrm{O}}$ \\
\hline 5845.0201 & .0201 & 5.00 & .018 & -1.18 & 5093.250 & 8.0 & 1.247 & 22197.090 & 9.0 & 1.218 & $\left({ }^{4} \mathrm{I}\right)^{5} \mathrm{~K}^{\mathrm{o}}$ \\
\hline 5851.5419R & & & .533 & -1.55 & 3714.537 & 7.0 & 1.177 & 20799.314 & 6.0 & 1.021 & $\left({ }^{4} \mathrm{I}\right)^{3} \mathrm{I}^{\mathrm{O}}$ \\
\hline
\end{tabular}


T. Ryabchikova et al.: Rare-earth elements in the atmosphere of the magnetic chemically peculiar star HD 144897, Online Material p 3

Table 4. continued.

\begin{tabular}{|c|c|c|c|c|c|c|c|c|c|c|c|}
\hline \multicolumn{4}{|c|}{ Wavelength $(\AA)$} & & \multicolumn{3}{|c|}{ Lower level $^{a}$} & \multicolumn{4}{|c|}{ Upper level } \\
\hline This work $^{b}$ & $\exp ^{c}$ & $\exp ^{d}$ & Stellar ${ }^{e}$ & $\log (g f)$ & $E\left(\mathrm{~cm}^{-1}\right)$ & $J$ & $g$ & $E\left(\mathrm{~cm}^{-1}\right)$ & $J$ & $g$ & LS term \\
\hline 5987.6828 & .6828 & & .679 & -1.26 & 3714.537 & 7.0 & 1.177 & 20410.864 & 8.0 & 1.150 & $\left({ }^{4} I\right)^{5} \mathrm{~K}^{0}$ \\
\hline 6145.0677 & .0677 & .021 & .058 & -1.33 & 2387.529 & 6.0 & 1.071 & 18656.240 & 7.0 & 1.054 & $\left({ }^{4} \mathrm{I}\right)^{5} \mathrm{~K}^{\mathrm{o}}$ \\
\hline 6327.2649 & 2649 & .220 & .266 & -1.41 & 1137.795 & 5.0 & 0.902 & 16938.043 & 6.0 & 0.912 & $\left({ }^{4} \mathrm{I}\right)^{5} \mathrm{~K}^{\mathrm{o}}$ \\
\hline $6526.6288 \mathrm{R}$ & & & .638 & -2.51 & 5093.250 & 8.0 & 1.247 & 20410.864 & 8.0 & 1.150 & $\left({ }^{4} \mathrm{I}\right)^{5} \mathrm{~K}^{\mathrm{o}}$ \\
\hline 6550.2314 & .2314 & .212 & .237 & -1.49 & 0.000 & 4.0 & 0.605 & 15262.420 & 5.0 & 0.688 & $\left({ }^{4} \mathrm{I}\right)^{5} \mathrm{~K}^{\mathrm{o}}$ \\
\hline $6690.8302 R$ & & & .833 & -2.46 & 3714.537 & 7.0 & 1.177 & 18656.240 & 7.0 & 1.054 & $\left({ }^{4} \mathrm{I}\right)^{5} \mathrm{~K}^{\mathrm{o}}$ \\
\hline $6870.7137 \mathrm{R}$ & & & $.71 \mathrm{bl}$ & -2.58 & 2387.529 & 6.0 & 1.071 & 16938.043 & 6.0 & 0.912 & $\left({ }^{4} \mathrm{I}\right)^{5} \mathrm{~K}^{\mathrm{o}}$ \\
\hline $7077.8825 \mathrm{R}$ & & & .885 & -2.85 & 1137.795 & 5.0 & 0.902 & 15262.420 & 5.0 & 0.688 & $\left({ }^{4} \mathrm{I}\right)^{5} \mathrm{~K}^{\mathrm{o}}$ \\
\hline
\end{tabular}

${ }^{a}$ Lower levels belong to the $4 \mathrm{f}^{4}{ }^{5} \mathrm{I}$ term.

${ }^{b} \mathrm{R}$ - wavelengths calculated from known level energies (Ritz wavelengths).

${ }^{c}$ Aldenius (2001).

${ }^{d}$ Based on data provided by Crosswhite (1976).

${ }^{e}$ Measured in HD 217522 spectrum.

${ }^{f}$ Upper levels belong to the $4 \mathrm{f}^{3} 5 \mathrm{~d}$ configuration. See text for A,B.

Table 5. Line-by-line abundances in HD 144897.

\begin{tabular}{|c|c|c|c|}
\hline Wavelength $(\AA)$ & $E_{\text {low }}(\mathrm{eV})$ & $\log (g f)$ & $\log \left(N / N_{\text {tot }}\right)$ \\
\hline \multicolumn{4}{|l|}{ OI } \\
\hline 7771.9413 & 9.146 & 0.369 & -4.00 \\
\hline 7774.1607 & 9.146 & 0.223 & -4.00 \\
\hline 7775.3904 & 9.146 & 0.001 & -4.00 \\
\hline \multicolumn{4}{|l|}{ CoI } \\
\hline 3412.3330 & 0.514 & 0.030 & -4.80 \\
\hline 3502.2780 & 0.432 & 0.070 & -4.60 \\
\hline 3506.3120 & 0.514 & -0.040 & -4.60 \\
\hline \multicolumn{4}{|l|}{ Co II } \\
\hline 3415.7720 & 2.203 & -1.640 & -5.10 \\
\hline 3514.2140 & 2.274 & -2.970 & -4.20 \\
\hline 3517.9900 & 3.009 & -2.560 & -4.30 \\
\hline 3523.5460 & 2.729 & -1.540 & -4.80 \\
\hline 3550.7120 & 2.985 & -2.680 & -4.40 \\
\hline 3566.9590 & 2.729 & -2.440 & -4.70 \\
\hline 4915.4230 & 3.406 & -3.425 & -4.20 \\
\hline \multicolumn{4}{|l|}{ Sr II } \\
\hline 3380.7070 & 2.940 & 0.199 & -7.10 \\
\hline 4161.7920 & 2.940 & -0.502 & -7.00 : \\
\hline 4215.5190 & 0.000 & -0.145 & -8.20 \\
\hline 4305.4430 & 3.040 & -0.136 & -7.50 \\
\hline \multicolumn{4}{|l|}{ Y II } \\
\hline 3242.2800 & 0.180 & 0.210 & -7.75 \\
\hline 4900.1200 & 1.033 & -0.090 & -7.60 \\
\hline 5662.9250 & 1.944 & 0.160 & -7.75 \\
\hline \multicolumn{4}{|l|}{ Zr II } \\
\hline 3129.1530 & 0.527 & -0.320 & -7.30 \\
\hline 3129.7630 & 0.039 & -0.650 & -7.30 \\
\hline 3138.6830 & 0.095 & -0.460 & -7.30 \\
\hline 3241.0420 & 0.039 & -0.504 & -7.60 \\
\hline 3305.1530 & 0.039 & -0.590 & -7.20 \\
\hline 3344.7860 & 1.011 & -0.220 & -7.40 \\
\hline 3354.3900 & 0.758 & -0.744 & -7.30 \\
\hline 4149.2170 & 0.802 & -0.030 & -7.60 \\
\hline \multicolumn{4}{|l|}{ Ba II } \\
\hline 4554.0290 & 0.000 & 0.170 & -9.00 \\
\hline \multicolumn{4}{|l|}{ La II } \\
\hline 4123.2180 & 0.321 & 0.130 & -7.30 \\
\hline 4655.4800 & 1.946 & 0.115 & -7.40 \\
\hline 4748.7260 & 0.927 & -0.540 & -7.40 \\
\hline 4899.9150 & 0.000 & -0.730 & -7.60 \\
\hline \multicolumn{4}{|l|}{ Ce II } \\
\hline 4127.3640 & 0.684 & 0.350 & -6.80 \\
\hline 4142.3970 & 0.696 & 0.300 & -6.80 \\
\hline 4248.6710 & 0.684 & 0.140 & -6.40 \\
\hline
\end{tabular}


T. Ryabchikova et al.: Rare-earth elements in the atmosphere of the magnetic chemically peculiar star HD 144897, Online Material p 4

Table 5. continued.

\begin{tabular}{|c|c|c|c|}
\hline Wavelength $(\AA)$ & $E_{\text {low }}(\mathrm{eV})$ & $\log (g f)$ & $\log \left(N / N_{\mathrm{tot}}\right)$ \\
\hline 4460.2070 & 0.478 & 0.320 & -6.90 \\
\hline 4560.2800 & 0.910 & 0.310 & -6.80 \\
\hline 4562.3590 & 0.478 & 0.230 & -6.80 \\
\hline 4606.4000 & 0.910 & -0.020 & -6.40 \\
\hline 4680.1190 & 1.058 & -0.430 & -6.40 \\
\hline 4737.2710 & 1.090 & -0.040 & -6.80 \\
\hline 5079.6820 & 1.384 & 0.420 & -6.80 \\
\hline \multicolumn{4}{|l|}{ Ce III } \\
\hline 3085.0990 & 2.385 & 0.010 & -6.80 \\
\hline 3106.9800 & 2.413 & -0.200 & -6.50 \\
\hline 3353.2860 & 2.663 & 0.180 & -6.80 \\
\hline 3395.7700 & 2.709 & -0.500 & -6.40 \\
\hline 3427.3580 & 2.385 & -0.170 & -6.75 \\
\hline 3454.3870 & 2.413 & -0.060 & -6.70 \\
\hline 3470.9200 & 2.413 & 0.140 & -6.80 \\
\hline 4535.7260 & 2.663 & -1.600 & -6.40 \\
\hline \multicolumn{4}{|l|}{ Pr II } \\
\hline 4206.7190 & 0.550 & 0.480 & -6.70 \\
\hline 5322.7720 & 0.483 & -0.315 & -6.50 \\
\hline \multicolumn{4}{|l|}{ Pr III } \\
\hline 4725.5590 & 2.078 & -1.365 & -6.70 \\
\hline 4929.1150 & 0.359 & -2.068 & -6.70 \\
\hline 5284.6930 & 0.173 & -0.771 & -6.70 \\
\hline 5299.9930 & 0.359 & -0.520 & -6.50 \\
\hline 5844.4080 & 1.244 & -1.011 & -6.70 \\
\hline 5998.9300 & 0.173 & -1.872 & -6.70 \\
\hline 6090.0100 & 0.359 & -0.871 & -6.70 \\
\hline 6160.2330 & 0.173 & -1.020 & -6.70 \\
\hline 6195.6190 & 0.000 & -1.071 & -6.70 \\
\hline 6692.2467 & 1.162 & -2.111 & -6.50 \\
\hline 7030.3850 & 0.359 & -0.929 & -6.50 \\
\hline 7076.6120 & 0.173 & -1.429 & -6.50 \\
\hline 7781.9830 & 0.000 & -1.276 & -7.00 \\
\hline 7888.1236 & 1.346 & -1.267 & -7.00 \\
\hline \multicolumn{4}{|l|}{$\mathrm{Nd}$ II } \\
\hline 4061.0800 & 0.471 & 0.550 & -6.80 \\
\hline 4156.0780 & 0.182 & 0.160 & -6.50 \\
\hline 4462.9790 & 0.559 & 0.040 & -6.50 \\
\hline 4542.6000 & 0.742 & -0.280 & -6.50 \\
\hline 4645.7600 & 0.559 & -0.760 & -6.40 \\
\hline 4706.5430 & 0.000 & -0.710 & -6.50 \\
\hline 4715.5860 & 0.205 & -0.900 & -6.50 \\
\hline 4724.3570 & 0.742 & -0.430 & -6.50 \\
\hline 5319.8150 & 0.550 & -0.140 & -6.40 \\
\hline \multicolumn{4}{|c|}{ Nd III: previous classification } \\
\hline 4927.4880 & 0.461 & -0.800 & -6.75 \\
\hline 5286.7530 & 0.632 & -1.880 & -6.25 \\
\hline 5294.1130 & 0.000 & -0.700 & -6.55 \\
\hline 5264.9600 & 0.461 & -0.720 & -6.60 \\
\hline 5633.5540 & 0.141 & -2.220 & -6.60 \\
\hline 5677.1790 & 0.632 & -1.450 & -6.50 \\
\hline 5845.0200 & 0.632 & -1.180 & -6.50 \\
\hline 5987.6830 & 0.461 & -1.260 & -6.60 \\
\hline 6145.0680 & 0.296 & -1.340 & -6.50 \\
\hline 6327.2650 & 0.141 & -1.410 & -6.35 \\
\hline 6550.2310 & 0.000 & -1.490 & -6.50 \\
\hline 6690.8300 & 0.461 & -2.440 & -6.10 \\
\hline 7077.8830 & 0.141 & -2.840 & -6.10 \\
\hline \multicolumn{4}{|c|}{ Nd III: new and reclassified lines } \\
\hline 3442.7890 & 0.141 & -1.490 & -6.70 \\
\hline 3476.1890 & 0.296 & -2.000 & -6.50 \\
\hline 3477.8360 & 0.000 & -1.660 & -6.60 \\
\hline 3537.6120 & 0.141 & -2.600 & -6.10 \\
\hline 3590.3290 & 0.461 & -0.590 & -6.85 \\
\hline 3597.6280 & 0.296 & -0.570 & -6.50 \\
\hline 3612.3388 & 0.000 & -0.800 & -7.20 \\
\hline 3644.3530 & 0.460 & -0.850 & -6.60 \\
\hline
\end{tabular}


T. Ryabchikova et al.: Rare-earth elements in the atmosphere of the magnetic chemically peculiar star HD 144897, Online Material p 5

Table 5. continued.

\begin{tabular}{|c|c|c|c|}
\hline Wavelength $(\AA)$ & $E_{\text {low }}(\mathrm{eV})$ & $\log (g f)$ & $\log \left(N / N_{\mathrm{tot}}\right)$ \\
\hline 4414.2978 & 0.296 & -2.220 & -6.50 \\
\hline 4445.9970 & 0.296 & -2.220 & -6.60 \\
\hline 4466.3550 & 0.460 & -2.520 & -6.30 \\
\hline 4473.2920 & 0.631 & -1.300 & -6.90 \\
\hline 4570.6430 & 0.141 & -2.060 & -6.65 \\
\hline 4613.1780 & 0.296 & -3.140 & $-6.3:$ \\
\hline 4627.2540 & 0.460 & -2.080 & -6.55 \\
\hline 4651.6230 & 0.141 & -1.860 & -6.80 \\
\hline 4654.3115 & 0.000 & -1.920 & -6.80 \\
\hline 4689.0552 & 0.461 & -1.800 & -6.65 \\
\hline 4759.5260 & 0.631 & -1.150 & -6.70 \\
\hline 4769.6217 & 0.296 & -1.800 & -6.70 \\
\hline 4788.4620 & 0.460 & -1.770 & -6.70 \\
\hline 4796.5000 & 0.141 & -1.650 & -6.70 \\
\hline 4821.9860 & 0.296 & -2.450 & -6.40 \\
\hline 4903.2410 & 0.000 & -2.210 & -6.65 \\
\hline 4911.6530 & 0.141 & -1.630 & -6.75 \\
\hline 4912.9440 & 0.000 & -1.780 & -6.70 \\
\hline 4914.0940 & 0.460 & -1.100 & -6.70 \\
\hline 4942.6730 & 0.631 & -1.230 & -6.70 \\
\hline 5050.6920 & 0.296 & -1.060 & -6.30 \\
\hline 5084.6590 & 0.141 & -2.580 & -6.50 \\
\hline 5102.4380 & 0.296 & -0.620 & -6.20 \\
\hline 5127.0440 & 0.631 & -0.400 & -6.50 \\
\hline 5151.7310 & 0.460 & -1.540 & -6.65 \\
\hline 5193.0400 & 0.141 & -1.180 & -6.70 \\
\hline 5203.9240 & 0.141 & -0.660 & -6.70 \\
\hline 5410.0990 & 0.141 & -1.520 & -6.50 \\
\hline 5429.7940 & 0.296 & -1.240 & -6.60 \\
\hline 5566.0150 & 0.296 & -2.330 & -6.40 \\
\hline 5851.5420 & 0.460 & -1.550 & -6.60 \\
\hline \multicolumn{4}{|l|}{ Sm II } \\
\hline 3568.2710 & 0.485 & 0.290 & -7.20 \\
\hline 4420.5240 & 0.333 & -0.430 & -6.75 \\
\hline 4424.3370 & 0.485 & 0.140 & -6.86 \\
\hline 4467.3410 & 0.659 & 0.150 & -7.10 \\
\hline \multicolumn{4}{|l|}{ Sm III } \\
\hline 3269.3290 & 0.388 & -0.960 & -7.00 \\
\hline 3355.3370 & 0.388 & -1.510 & -7.00 \\
\hline 3392.2610 & 0.100 & -1.990 & -7.05 \\
\hline 3414.4750 & 0.282 & -1.370 & -7.00 \\
\hline 3433.5990 & 0.185 & -1.360 & -6.95 \\
\hline 3453.2090 & 0.100 & -1.520 & -7.00 \\
\hline 3491.3030 & 0.000 & -2.960 & -6.55 \\
\hline 3527.4520 & 0.036 & -1.920 & -7.20 \\
\hline 3528.7730 & 0.282 & -2.040 & -6.60 \\
\hline 3536.1650 & 0.185 & -1.900 & -6.80 \\
\hline \multicolumn{4}{|l|}{ Gd II } \\
\hline 3330.3390 & 0.991 & 0.119 & -7.00 \\
\hline 3360.7120 & 0.032 & -0.240 & -7.10 \\
\hline 3362.2390 & 0.079 & 0.294 & -7.10 \\
\hline 4049.4230 & 0.662 & -0.124 & -7.10 \\
\hline 4049.8550 & 0.991 & 0.429 & -7.20 \\
\hline 4130.3660 & 0.731 & -0.090 & -7.00 \\
\hline 4131.4730 & 1.406 & -0.105 & -7.00 \\
\hline 4162.7330 & 0.492 & -0.720 & -7.10 \\
\hline 4163.0870 & 0.662 & -0.837 & -7.00 \\
\hline 4184.2580 & 0.492 & -0.080 & -6.70 \\
\hline 4215.0220 & 0.427 & -0.550 & -6.70 \\
\hline 4732.6090 & 1.102 & -0.695 & -6.70 \\
\hline 4755.3430 & 2.449 & -0.235 & -6.70 \\
\hline \multicolumn{4}{|l|}{ Gd III } \\
\hline 3118.0410 & 1.432 & 0.000 & -6.70 \\
\hline 3176.6610 & 1.432 & -0.540 & -6.50 \\
\hline \multicolumn{4}{|l|}{ Tb II } \\
\hline 3509.1440 & 0.000 & 0.700 & -7.80 \\
\hline 3567.3490 & 0.439 & 0.190 & -7.80 \\
\hline
\end{tabular}


T. Ryabchikova et al.: Rare-earth elements in the atmosphere of the magnetic chemically peculiar star HD 144897, Online Material p 6

Table 5. continued.

\begin{tabular}{|c|c|c|c|}
\hline Wavelength $(\AA)$ & $E_{\text {low }}(\mathrm{eV})$ & $\log (g f)$ & $\log \left(N / N_{\mathrm{tot}}\right)$ \\
\hline 3568.5100 & 0.000 & 0.360 & -7.90 \\
\hline \multicolumn{4}{|l|}{ Tb III } \\
\hline 4774.1209 & 0.000 & -1.412 & -8.20 \\
\hline 5505.4080 & 0.000 & -0.815 & -8.10 \\
\hline 5847.2310 & 0.348 & -1.013 & -8.00 \\
\hline 6092.8960 & 0.587 & -1.133 & -7.80 \\
\hline 6323.6190 & 0.776 & -1.209 & -7.80 \\
\hline 6687.6980 & 1.027 & -1.355 & -7.60 \\
\hline \multicolumn{4}{|l|}{ Dy II } \\
\hline 3215.1920 & 1.662 & 0.780 & -7.20 \\
\hline 3407.7960 & 0.000 & 0.150 & -7.35 \\
\hline 3434.3690 & 0.000 & -0.430 & $-6.60:$ \\
\hline 3460.9690 & 0.000 & -0.160 & -7.00 \\
\hline 3487.6110 & 2.500 & 0.980 & -7.00 \\
\hline 3523.9830 & 0.538 & 0.430 & -7.10 \\
\hline 3531.7070 & 0.000 & 0.790 & -7.30 \\
\hline 3538.5190 & 0.000 & -0.190 & -7.00 \\
\hline 3550.2180 & 0.590 & 0.463 & -7.30 \\
\hline 3574.1530 & 0.925 & 0.284 & -7.30 \\
\hline 3595.0340 & 1.314 & 0.760 & -7.20 \\
\hline \multicolumn{4}{|l|}{ Eu II } \\
\hline 4129.7250 & 0.000 & 0.173 & -8.00 \\
\hline 4205.0420 & 0.000 & 0.120 & -7.80 \\
\hline 6437.6400 & 1.320 & -0.273 & -7.50 \\
\hline 6645.1200 & 1.380 & 0.205 & -7.40 \\
\hline \multicolumn{4}{|l|}{ Eu III } \\
\hline 6666.3470 & 3.977 & -1.470 & -6.40 \\
\hline 6772.2399 & 4.005 & -2.840 & -6.00 \\
\hline 6976.0288 & 3.496 & -2.280 & $-6.3:$ \\
\hline 7221.8380 & 4.006 & -1.560 & -6.60 \\
\hline 7750.5974 & 4.316 & -2.170 & -6.10 \\
\hline 8379.1830 & 3.961 & -1.850 & -6.50 \\
\hline \multicolumn{4}{|l|}{ Dy III } \\
\hline 3078.0560 & 4.098 & -0.490 & -6.50 \\
\hline 3085.9810 & 3.836 & -0.600 & -6.50 \\
\hline 3369.6550 & 0.000 & -1.110 & -7.20 \\
\hline 4401.5670 & 0.881 & -1.430 & -7.40 \\
\hline 4510.0270 & 0.881 & -1.850 & -7.15 \\
\hline 4572.8860 & 0.000 & -1.720 & -7.20 \\
\hline \multicolumn{4}{|l|}{ Ho II } \\
\hline 3416.4440 & 0.079 & 0.260 & -7.90 \\
\hline 3456.0100 & 0.000 & 0.760 & -8.10 \\
\hline 3484.8300 & 0.079 & 0.280 & -8.00 \\
\hline \multicolumn{4}{|l|}{ Ho III } \\
\hline 3435.2690 & 1.072 & -0.970 & -7.80 \\
\hline 3581.4470 & 0.000 & -0.890 & -8.00 \\
\hline 4267.0650 & 0.674 & -1.440 & -8.20 \\
\hline 4416.1360 & 0.000 & -1.550 & -8.15 \\
\hline 4448.1040 & 0.674 & -1.180 & -8.15 \\
\hline 4494.5230 & 0.000 & -1.360 & -8.20 \\
\hline \multicolumn{4}{|l|}{ Er II } \\
\hline 3303.9530 & 1.701 & 0.723 & -7.45 \\
\hline 3499.1030 & 0.055 & 0.139 & -7.65 \\
\hline \multicolumn{4}{|l|}{ Er III } \\
\hline 3070.4020 & 0.000 & -1.830 & -7.25 \\
\hline 3073.5360 & 0.630 & -1.640 & -7.10 \\
\hline 3469.0070 & 0.000 & -1.660 & -7.30 \\
\hline 4422.3140 & 0.000 & -1.740 & -7.40 \\
\hline 4540.7120 & 0.000 & -2.540 & -7.00 \\
\hline 4735.5540 & 0.630 & -1.580 & -7.30 \\
\hline 4783.1150 & 0.864 & -2.010 & -7.10 \\
\hline \multicolumn{4}{|l|}{ Tm II } \\
\hline 3267.3970 & 1.111 & 0.454 & -7.90 \\
\hline 3309.8010 & 1.111 & 0.423 & $\leq-8.3$ \\
\hline 3462.1970 & 0.000 & 0.030 & -8.15 \\
\hline \multicolumn{4}{|l|}{ Tm III } \\
\hline 3098.6370 & 0.000 & -2.250 & -7.50 \\
\hline
\end{tabular}


T. Ryabchikova et al.: Rare-earth elements in the atmosphere of the magnetic chemically peculiar star HD 144897, Online Material p 7

Table 5. continued.

\begin{tabular}{cccl}
\hline \hline Wavelength $(\AA)$ & $E_{\text {low }}(\mathrm{eV})$ & $\log (g f)$ & $\log \left(N / N_{\text {tot }}\right)$ \\
\hline 3273.9130 & 0.000 & -1.460 & -7.90 \\
3629.0920 & 0.000 & -1.960 & -7.70 \\
Yb II & & & \\
$\quad 5335.1590$ & 3.789 & -0.260 & $-7.50:$ \\
Yb III & & & \\
$\quad 3092.4960$ & 4.979 & -0.370 & -7.50 \\
Lu II & & & \\
$\quad 6221.8600$ & 1.541 & -0.760 & -8.60 \\
\hline
\end{tabular}


T. Ryabchikova et al.: Rare-earth elements in the atmosphere of the magnetic chemically peculiar star HD 144897, Online Material p 8

Table 6. Fitted (FIT) and Hartree-Fock (HF) energy parameters $\left(\mathrm{cm}^{-1}\right)$ and their ratios for the $4 \mathrm{f}^{4}$ and $4 \mathrm{f}^{3} 5 \mathrm{~d}$ configurations of Nd III.

\begin{tabular}{|c|c|c|c|c|}
\hline Parameter & $\overline{\text { FIT }}$ & $\overline{\mathrm{HF}}$ & ratio & $\overline{\text { Remark }}$ \\
\hline$E_{a v}\left(4 \mathrm{f}^{4}\right)$ & $31510(0)$ & 41429 & & \\
\hline$F^{2}(4 f, 4 f)$ & 66417 & 92632 & 0.717 & $\mathrm{f}$ \\
\hline $\mathrm{F}^{4}(4 \mathrm{f}, 4 \mathrm{f})$ & 45796 & 57678 & 0.794 & $\mathrm{f}$ \\
\hline$F^{6}(4 f, 4 f)$ & 31773 & 41370 & 0.768 & $\mathrm{f}$ \\
\hline$\alpha$ & 30 & & & $\mathrm{f}$ \\
\hline$\beta$ & -823 & & & $\mathrm{f}$ \\
\hline$\gamma$ & 1268 & & & f \\
\hline$\zeta(4 \mathrm{f})$ & $774(0)$ & 849 & 0.912 & \\
\hline$\sigma$ & 1 & & & \\
\hline$E_{a v}\left(4 \mathrm{f}^{3} 5 \mathrm{~d}\right)$ & $44696(63)$ & 42684 & & \\
\hline$F^{2}(4 f, 4 f)$ & $75830(211)$ & 101374 & 0.748 & r1 \\
\hline$F^{4}(4 f, 4 f)$ & $52641(146)$ & 63549 & 0.828 & r1 \\
\hline $\mathrm{F}^{6}(4 \mathrm{f}, 4 \mathrm{f})$ & $36618(102)$ & 45703 & 0.801 & r1 \\
\hline$\alpha$ & 30 & & & $\mathrm{f}$ \\
\hline$\beta$ & -823 & & & $\mathrm{f}$ \\
\hline$\gamma$ & 1268 & & & $\mathrm{f}$ \\
\hline$\zeta(4 \mathrm{f})$ & $890(5)$ & 946 & 0.940 & \\
\hline$\zeta(5 d)$ & $815(14)$ & 830 & 0.981 & \\
\hline $\mathrm{F}^{1}(4 \mathrm{f}, 5 \mathrm{~d})$ & 1200 & & & $f$ \\
\hline$F^{2}(4 f, 5 d)$ & $20656(363)$ & 26407 & 0.782 & r2 \\
\hline $\mathrm{F}^{4}(4 \mathrm{f}, 5 \mathrm{~d})$ & $10185(179)$ & 13021 & 0.782 & r2 \\
\hline $\mathrm{G}^{1}(4 \mathrm{f}, 5 \mathrm{~d})$ & $9168(82)$ & 12823 & 0.715 & r3 \\
\hline$G^{3}(4 f, 5 d)$ & $7294(65)$ & 10202 & 0.715 & r3 \\
\hline$G^{5}(4 f, 5 d)$ & $5530(50)$ & 7734 & 0.715 & r3 \\
\hline$\sigma$ & 55 & & & \\
\hline
\end{tabular}

$\mathrm{f}$ - parameter is fixed.

$\mathrm{r} 1$ - parameter ratios are fixed on the same values as in $4 \mathrm{f}^{4}$.

$\mathrm{r} 2, \mathrm{r} 3$ - parameter ratios are fixed on HF values. 
T. Ryabchikova et al.: Rare-earth elements in the atmosphere of the magnetic chemically peculiar star HD 144897, Online Material p 9

Table 7. Energy levels $\left(\mathrm{cm}^{-1}\right)$ of the $4 \mathrm{f}^{3} 5 \mathrm{~d}(J=3-10)$ configuration of $\mathrm{Nd}$ III below $33000 \mathrm{~cm}^{-1}$.

\begin{tabular}{|c|c|c|c|c|}
\hline$E^{a}$ & $\Delta E^{b}$ & $g$ & Composition $^{c}$ & Remark $^{d}$ \\
\hline \multicolumn{5}{|l|}{$J=3$} \\
\hline 19403.433 & -183 & 0.575 & $67 \%\left({ }^{4} \mathrm{I}\right){ }^{5} \mathrm{H}+18 \%\left({ }^{4} \mathrm{I}\right)^{3} \mathrm{G}+6 \%\left({ }^{4} \mathrm{~F}\right)^{5} \mathrm{H}$ & \\
\hline 21479.437 & 48 & 0.825 & $60 \%\left({ }^{4} \mathrm{I}\right)^{5} \mathrm{G}+18 \%\left({ }^{4} \mathrm{I}\right)^{3} \mathrm{G}+15 \%\left({ }^{4} \mathrm{I}\right)^{5} \mathrm{H}$ & \\
\hline$(24094)$ & & 0.789 & $56 \%\left({ }^{4} \mathrm{I}\right)^{3} \mathrm{G}+30 \%\left({ }^{4} \mathrm{I}\right)^{5} \mathrm{G}+7 \%\left({ }^{4} \mathrm{I}\right)^{5} \mathrm{H}$ & \\
\hline 27675.003 & 45 & 0.836 & $51 \%\left({ }^{4} \mathrm{~F}\right){ }^{5} \mathrm{H}+10 \%\left({ }^{4} \mathrm{~F}\right){ }^{5} \mathrm{D}+9 \%\left({ }^{4} \mathrm{~S}\right){ }^{5} \mathrm{D}$ & \\
\hline$(27851)$ & & 1.072 & $33 \%\left({ }^{4} \mathrm{~F}\right){ }^{5} \mathrm{H}+20 \%\left({ }^{4} \mathrm{~F}\right){ }^{5} \mathrm{D}+17 \%\left({ }^{4} \mathrm{~S}\right)^{5} \mathrm{D}$ & \\
\hline$(29436)$ & & 0.952 & $81 \%\left({ }^{4} \mathrm{~F}\right)^{5} \mathrm{G}+7 \%\left({ }^{4} \mathrm{~F}\right)^{3} \mathrm{~F}+2 \%\left({ }_{1}^{2} \mathrm{D}\right)^{3} \mathrm{~F}$ & \\
\hline$(30765)$ & & 1.219 & $14 \%\left({ }^{4} \mathrm{~S}\right){ }^{5} \mathrm{D}+11 \%\left({ }_{2}^{2} \mathrm{H}\right){ }^{1} \mathrm{~F}+11 \%\left({ }_{2}^{2} \mathrm{H}\right)^{3} \mathrm{~F}$ & \\
\hline (31 159) & & 1.160 & $22 \%\left({ }^{4} \mathrm{~F}\right){ }^{3} \mathrm{~F}+18 \%\left({ }^{4} \mathrm{G}\right){ }^{3} \mathrm{~F}+17 \%\left({ }^{4} \mathrm{~F}\right){ }^{5} \mathrm{P}$ & \\
\hline (31 572) & & 1.313 & $31 \%\left({ }^{4} \mathrm{~F}\right){ }^{5} \mathrm{P}+22 \%\left({ }^{4} \mathrm{~F}\right){ }^{3} \mathrm{D}+9 \%\left({ }^{4} \mathrm{~S}\right)^{3} \mathrm{D}$ & \\
\hline$(32438)$ & & 1.233 & $47 \%\left({ }^{4} \mathrm{~F}\right)^{5} \mathrm{~F}+10 \%\left({ }^{4} \mathrm{~S}\right)^{3} \mathrm{D}+7 \%\left({ }^{4} \mathrm{~F}\right)^{5} \mathrm{P}$ & \\
\hline$(32543)$ & & 1.217 & $29 \%\left({ }^{4} \mathrm{~F}\right)^{5} \mathrm{~F}+24 \%\left({ }^{4} \mathrm{~F}\right)^{5} \mathrm{P}+10 \%\left({ }_{2}^{2} \mathrm{H}\right)^{3} \mathrm{G}$ & \\
\hline \multicolumn{5}{|l|}{$J=4$} \\
\hline 18883.652 & 74 & $\overline{0.640}$ & $81 \%\left({ }^{4} \mathrm{I}\right)^{5} \mathrm{I}+15 \%\left({ }^{4} \mathrm{I}\right)^{3} \mathrm{H}+1 \%\left({ }^{4} \mathrm{I}\right)^{5} \mathrm{H}$ & \\
\hline 20388.980 & -11 & 0.836 & $46 \%\left({ }^{4} \mathrm{I}\right){ }^{5} \mathrm{H}+28 \%\left({ }^{4} \mathrm{I}\right)^{3} \mathrm{H}+11 \%\left({ }^{4} \mathrm{I}\right)^{5} \mathrm{I}$ & \\
\hline 21491.858 & 26 & 0.948 & $19 \%\left({ }^{4} \mathrm{I}\right)^{3} \mathrm{H}+29 \%\left({ }^{4} \mathrm{I}\right)^{5} \mathrm{H}+20 \%\left({ }^{4} \mathrm{I}\right)^{5} \mathrm{G}$ & \\
\hline 23010.343 & 36 & 1.031 & $56 \%\left({ }^{4} \mathrm{I}\right){ }^{5} \mathrm{G}+18 \%\left({ }^{4} \mathrm{I}\right)^{3} \mathrm{H}+11 \%\left({ }^{4} \mathrm{I}\right)^{5} \mathrm{H}$ & \\
\hline$(26481)$ & & 1.057 & $71 \%\left({ }^{4} \mathrm{I}\right)^{3} \mathrm{G}+17 \%\left({ }^{4} \mathrm{I}\right)^{5} \mathrm{G}+4 \%\left({ }^{4} \mathrm{I}\right){ }^{5} \mathrm{H}$ & \\
\hline 28745.285 & -43 & 0.916 & $83 \%\left({ }^{4} \mathrm{~F}\right)^{5} \mathrm{H}+7 \%\left({ }^{4} \mathrm{I}\right)^{5} \mathrm{H}+2 \%\left({ }_{1}^{2} \mathrm{D}\right)^{3} \mathrm{G}$ & \\
\hline (29 113) & & 1.421 & $38 \%\left({ }^{4} \mathrm{~F}\right)^{5} \mathrm{D}+32 \%\left({ }^{4} \mathrm{~S}\right){ }^{5} \mathrm{D}+7 \%\left({ }^{4} \mathrm{G}\right)^{5} \mathrm{D}$ & \\
\hline (30 252) & & 1.075 & $62 \%\left({ }^{4} \mathrm{~F}\right){ }^{5} \mathrm{G}+10 \%\left({ }^{4} \mathrm{~F}\right)^{3} \mathrm{H}+5 \%\left({ }_{1}^{2} \mathrm{G}\right)^{3} \mathrm{H}$ & \\
\hline (31 060) & & 0.928 & $21 \%\left({ }_{2}^{2} \mathrm{H}\right)^{3} \mathrm{H}+18 \%\left({ }^{4} \mathrm{~F}\right)^{5} \mathrm{G}+18 \%\left({ }^{4} \mathrm{~F}\right)^{3} \mathrm{H}$ & \\
\hline (31 932) & & 0.969 & $15 \%\left({ }_{2}^{2} \mathrm{H}\right) 1 \mathrm{G}+14 \%\left({ }_{2}^{2} \mathrm{H}\right)^{3} \mathrm{G}+9 \%\left({ }^{4} \mathrm{~F}\right)^{3} \mathrm{H}$ & \\
\hline$(32640)$ & & 0.719 & $69 \%\left({ }^{4} \mathrm{G}\right)^{5} \mathrm{I}+4 \%\left({ }_{2}^{2} \mathrm{H}\right)^{3} \mathrm{G}+3 \%\left({ }_{2}^{2} \mathrm{H}\right)^{3} \mathrm{H}$ & \\
\hline \multicolumn{5}{|l|}{$J=5$} \\
\hline 15262.420 & 28 & 0.688 & $88 \%\left({ }^{4} \mathrm{I}\right)^{5} \mathrm{~K}+8 \%\left({ }^{4} \mathrm{I}\right)^{3} \mathrm{I}+3 \%\left({ }_{2}^{2} \mathrm{H}\right)^{3} \mathrm{I}$ & \\
\hline 19616.608 & -69 & 0.869 & $53 \%\left({ }^{4} I\right)^{3} I+15 \%\left({ }^{4} I\right)^{5} I+9 \%\left({ }^{4} I\right)^{5} K$ & \\
\hline 20348.715 & 29 & 0.924 & $76 \%\left({ }^{4} \mathrm{I}\right)^{5} \mathrm{I}+12 \%\left({ }^{4} \mathrm{I}\right)^{3} \mathrm{H}+3 \%\left({ }^{4} \mathrm{I}\right)^{3} \mathrm{I}$ & \\
\hline 22181.265 & -8 & 1.032 & $58 \%\left({ }^{4} \mathrm{I}\right)^{5} \mathrm{H}+17 \%\left({ }^{4} \mathrm{I}\right)^{3} \mathrm{I}+8 \%\left({ }^{4} \mathrm{I}\right)^{3} \mathrm{H}$ & \\
\hline 23347.692 & 61 & 1.153 & $41 \%\left({ }^{4} \mathrm{I}\right){ }^{5} \mathrm{G}+18 \%\left({ }^{4} \mathrm{I}\right)^{3} \mathrm{H}+17 \%\left({ }^{4} \mathrm{I}\right)^{5} \mathrm{H}$ & \\
\hline 24878.419 & -12 & 1.141 & $32 \%\left({ }^{4} \mathrm{I}\right)^{3} \mathrm{H}+45 \%\left({ }^{4} \mathrm{I}\right)^{5} \mathrm{G}+7 \%\left({ }^{4} \mathrm{I}\right){ }^{5} \mathrm{H}$ & \\
\hline (28 927) & & 1.192 & $80 \%\left({ }^{4} \mathrm{I}\right)^{3} \mathrm{G}+7 \%\left({ }^{4} \mathrm{I}\right)^{5} \mathrm{G}+2 \%\left({ }^{4} \mathrm{~F}\right)^{3} \mathrm{G}$ & \\
\hline 29397.378 & -15 & 0.982 & $22 \%\left({ }^{4} \mathrm{~F}\right)^{5} \mathrm{H}+19 \%\left({ }_{2}^{2} \mathrm{H}\right)^{3} \mathrm{I}+7 \%\left({ }_{1}^{2} \mathrm{G}\right)^{3} \mathrm{I}$ & A \\
\hline 30175.690 & -7 & 1.051 & $60 \%\left({ }^{4} \mathrm{~F}\right){ }^{5} \mathrm{H}+7 \%\left({ }_{2}^{2} \mathrm{H}\right)^{3} \mathrm{I}+5 \%\left({ }^{4} \mathrm{I}\right)^{5} \mathrm{I} \quad 6 s$ & B \\
\hline$(31471)$ & & 1.135 & $59 \%\left({ }^{4} \mathrm{~F}\right)^{5} \mathrm{G}+13 \%\left({ }_{2}^{2} \mathrm{H}\right)^{3} \mathrm{I}+5 \%\left({ }^{4} \mathrm{~F}\right)^{3} \mathrm{H}$ & \\
\hline \multicolumn{5}{|l|}{$J=6$} \\
\hline$(15332)$ & & 0.725 & $93 \%\left({ }^{4} \mathrm{I}\right)^{5} \mathrm{~L}+3 \%\left({ }_{2}^{2} \mathrm{H}\right)^{3} \mathrm{~K}+3 \%\left({ }^{4} \mathrm{I}\right)^{3} \mathrm{~K}$ & \\
\hline 16938.043 & 25 & 0.912 & $94 \%\left({ }^{4} \mathrm{I}\right)^{5} \mathrm{~K}+4 \%\left({ }^{4} \mathrm{I}\right)^{3} \mathrm{I}+2 \%\left({ }_{2}^{2} \mathrm{H}\right)^{3} \mathrm{I}$ & \\
\hline 20799.314 & -10 & 1.021 & $39 \%\left({ }^{4} I\right)^{3} I+22 \%\left({ }^{4} I\right)^{5} I+13 \%\left({ }^{4} I\right)^{3} K$ & \\
\hline 21980.505 & -13 & 1.084 & $70 \%\left({ }^{4} \mathrm{I}\right)^{5} \mathrm{I}+8 \%\left({ }^{4} \mathrm{I}\right)^{3} \mathrm{H}+8 \%\left({ }^{4} \mathrm{I}\right)^{3} \mathrm{I}$ & \\
\hline 23120.081 & 52 & 0.940 & $53 \%\left({ }^{4} \mathrm{I}\right)^{3} \mathrm{~K}+14 \%\left({ }^{4} \mathrm{I}\right)^{5} \mathrm{H}+9 \%\left({ }_{2}^{2} \mathrm{H}\right)^{3} \mathrm{~K}$ & \\
\hline 24058.489 & -50 & 1.122 & $50 \%\left({ }^{4} \mathrm{I}\right)^{5} \mathrm{H}+28 \%\left({ }^{4} \mathrm{I}\right)^{3} \mathrm{I}+5 \%\left({ }^{4} \mathrm{I}\right)^{3} \mathrm{~K}$ & \\
\hline 25034.830 & 29 & 1.250 & $48 \%\left({ }^{4} \mathrm{I}\right){ }^{5} \mathrm{G}+22 \%\left({ }^{4} \mathrm{I}\right)^{3} \mathrm{H}+13 \%\left({ }^{4} \mathrm{I}\right){ }^{5} \mathrm{H}$ & \\
\hline$(26724)$ & & 1.234 & $43 \%\left({ }^{4} \mathrm{I}\right)^{5} \mathrm{G}+29 \%\left({ }^{4} \mathrm{I}\right)^{3} \mathrm{H}+6 \%\left({ }^{4} \mathrm{G}\right)^{3} \mathrm{H}$ & \\
\hline$(29509)$ & & 1.002 & $19 \%\left({ }_{2}^{2} \mathrm{H}\right)^{3} \mathrm{~K}+15 \%\left({ }^{4} \mathrm{I}\right)^{3} \mathrm{~K}+9 \%\left({ }_{2}^{2} \mathrm{H}\right)^{1} \mathrm{I}$ & \\
\hline 31146.429 & -30 & 1.060 & $31 \%\left({ }^{4} \mathrm{~F}\right)^{5} \mathrm{H}+31 \%\left({ }_{2}^{2} \mathrm{H}\right)^{3} \mathrm{~K}+8 \%\left({ }^{4} \mathrm{~F}\right)^{5} \mathrm{G}$ & A \\
\hline 31559.191 & 132 & 1.151 & $43 \%\left({ }^{4} \mathrm{~F}\right)^{5} \mathrm{H}+11 \%\left({ }^{4} \mathrm{~F}\right)^{5} \mathrm{G}+10 \%\left({ }_{2}^{2} \mathrm{H}\right)^{1} \mathrm{I}$ & B \\
\hline$(32696)$ & & 1.096 & $24 \%\left({ }_{2}^{2} \mathrm{H}\right)^{1} \mathrm{I}+23 \%\left({ }^{4} \mathrm{~F}\right)^{5} \mathrm{G}+12 \%\left({ }_{2}^{2} \mathrm{H}\right)^{3} \mathrm{I}$ & \\
\hline$(17150)$ & & 0.915 & $96 \%\left({ }^{4} \mathrm{I}\right)^{5} \mathrm{~L}+2 \%\left({ }_{2}^{2} \mathrm{H}\right)^{3} \mathrm{~K}+1 \%\left({ }^{4} \mathrm{I}\right)^{3} \mathrm{~K}$ & \\
\hline 18656.240 & 12 & 1.054 & $97 \%\left({ }^{4} \mathrm{I}\right)^{5} \mathrm{~K}+1 \%\left({ }^{4} \mathrm{I}\right)^{3} \mathrm{I}+1 \%\left({ }_{2}^{2} \mathrm{H}\right)^{3} \mathrm{I}$ & \\
\hline 22702.749 & -13 & 1.146 & $54 \%\left({ }^{4} \mathrm{I}\right)^{5} \mathrm{I}+22 \%\left({ }^{4} \mathrm{I}\right)^{3} \mathrm{I}+14 \%\left({ }^{4} \mathrm{I}\right)^{3} \mathrm{~K}$ & A \\
\hline 24003.190 & -12 & 1.176 & $35 \%\left({ }^{4} I\right)^{5} \mathrm{I}+32 \%\left({ }^{4} \mathrm{I}\right)^{3} \mathrm{I}+19 \%\left({ }^{4} \mathrm{I}\right)^{5} \mathrm{H}$ & B \\
\hline$(24602)$ & & 0.891 & $81 \%\left({ }^{4} \mathrm{I}\right)^{3} \mathrm{~L}+8 \%\left({ }^{2} \mathrm{~K}\right)^{3} \mathrm{~L}+3 \%\left({ }^{4} \mathrm{I}\right)^{3} \mathrm{~K}$ & \\
\hline 25319.572 & 57 & 1.099 & $49 \%\left({ }^{4} \mathrm{I}\right)^{3} \mathrm{~K}+26 \%\left({ }^{4} \mathrm{I}\right)^{5} \mathrm{H}+9 \%\left({ }_{2}^{2} \mathrm{H}\right)^{3} \mathrm{~K}$ & \\
\hline 26097.872 & -63 & 1.186 & $42 \%\left({ }^{4} \mathrm{I}\right){ }^{5} \mathrm{H}+31 \%\left({ }^{4} \mathrm{I}\right)^{3} \mathrm{I}+12 \%\left({ }^{4} \mathrm{I}\right)^{3} \mathrm{~K}$ & \\
\hline 31781.776 & -52 & 1.141 & $33 \%\left({ }^{4} \mathrm{~F}\right)^{5} \mathrm{H}+27 \%\left({ }_{2}^{2} \mathrm{H}\right)^{3} \mathrm{~K}+9 \%\left({ }_{1}^{2} \mathrm{G}\right)^{3} \mathrm{I}$ & A \\
\hline 32832.419 & -39 & 1.155 & $45 \%\left({ }^{4} \mathrm{~F}\right)^{5} \mathrm{H}+30 \%\left({ }_{2}^{2} \mathrm{H}\right)^{3} \mathrm{~K}+6 \%\left({ }^{2} \mathrm{~K}\right)^{3} \mathrm{~K}$ & B \\
\hline \multicolumn{5}{|l|}{$J=8$} \\
\hline$(19082)$ & & 1.043 & $99 \%\left({ }^{4} \mathrm{I}\right)^{5} \mathrm{~L}+1 \%\left({ }_{2}^{2} \mathrm{H}\right)^{3} \mathrm{~K}$ & \\
\hline 20410.864 & -06 & 1.150 & $\left.97 \%\left({ }^{4} \mathrm{I}\right)\right)^{5} \mathrm{~K}+1 \%\left({ }^{4} \mathrm{I}\right){ }^{3} \mathrm{~L}+1 \%\left({ }^{2} \mathrm{~K}\right)^{3} \mathrm{~L}$ & \\
\hline 24592.234 & -67 & 1.229 & $83 \%\left({ }^{4} \mathrm{I}\right)^{5} \mathrm{I}+14 \%\left({ }^{4} \mathrm{I}\right)^{3} \mathrm{~K}+1 \%\left({ }^{2} \mathrm{~K}\right)^{3} \mathrm{~K}$ & \\
\hline$(26769)$ & & 1.019 & $84 \%\left({ }^{4} I\right)^{3} \mathrm{~L}+8 \%\left({ }^{2} \mathrm{~K}\right)^{3} \mathrm{~L}+2 \%\left({ }^{4} \mathrm{I}\right)^{5} \mathrm{~K}$ & \\
\hline 27441.879 & 77 & 1.140 & $67 \%\left({ }^{4} \mathrm{I}\right)^{3} \mathrm{~K}+13 \%\left({ }^{4} \mathrm{I}\right)^{5} \mathrm{I}+12 \%\left({ }_{2}^{2} \mathrm{H}\right)^{3} \mathrm{~K}$ & \\
\hline
\end{tabular}


T. Ryabchikova et al.: Rare-earth elements in the atmosphere of the magnetic chemically peculiar star HD 144897, Online Material p 10

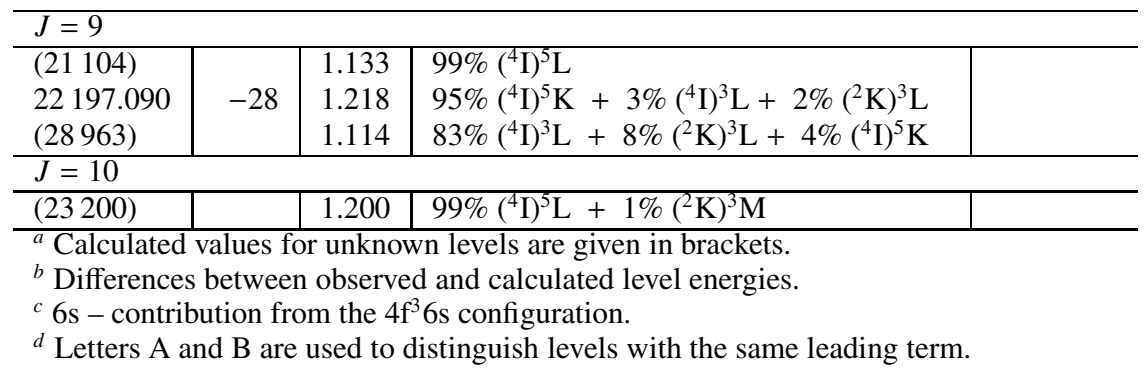

